\title{
A Holistic view of the biology of pancreatic cancer and targeted therapeutic approaches to treat the cancer
}

Sidra Munir, ${ }^{* 1}$ Shabir Ahmad, ${ }^{* 1}$ Ammara Gul ${ }^{1}$

Corresponding author: Sidra Munir, Department of Chemistry, Islamia college university Peshawar, Pakistan Postal code 25000

Corresponding author:, Department of Chemistry, Dr. Shabir Ahmad, Associate Professor, Islamia college university Peshawar, Pakistan, Postal code 25000

\begin{abstract}
Pancreatic cancer is a fatal disease with mortality rate of $5 \%$ and a with a limited survival rate of 5 years. Despite of the extensive efforts that has been made to cure the disease it still has been considered as 'undruggable'. It is characterized by epithelial to dense stromal tumor formation however due to lack of diagnosis options and treatment test available to detect the disease to the point at which resection of the tumor is possible, it makes it the fourth leading cause of cancer related death. The unavailable information regarding the early detection of biological markers along with the increased invasive tumor, the inherit chemoresistance against medication radiation and chemotherapy stubbornly fail the therapeutic options available. These associated problems made the scientists to reevaluate the approaches that are currently in practice and take a step back to fully understand the basic biological pathways that are involved in the pancreatic cancer, the heterogeneity of the tumor itself along with expression and a number of mutations that are observed at different locations. Clinical trial along with new approaches are nowadays focus of research to treat this tumor. The review paper describes the basic cellular pathways involved in pancreatic cancer, the gene mutations and their expression having effect on the pathology of the diseases along with treatment options that are available to treat the tumor. These efforts will help with the expansion of our knowledge to undergo the clinical trial and the synthesis of novel medicines for the prognosis of the disease.
\end{abstract}

Keywords: PDAC, Nrf2, K-Ras, ROS 


\section{Introduction}

Cancer induced death has increased to an alarming rate over the past decade despite of the immense efforts that has been carried out over the decade for its effective treatment it still has been marked by poor diagnosis and low survival rate. Novel approaches from radiation chemotherapy to selective target drugs have been developed but none has the ability to classify it as a nonmalignant disease. [13] The therapeutic approaches to treat cancer includes surgery, [14, 15] radiation therapy, [16] chemotherapy, [17, 18] Drug therapy [19, 20], photodynamic therapy [21] etc. If the tumor is resectable then chemo or radiation therapy is afterwards recommended to avoid reoccurrence. Endoscopically pancreatic juice introduced into the patients having signs and symptoms of pancreatic disease has been suggested to determine the early detection of PC as the cancer once progresses has less chances of survival. [22]

Pancreatic cancer has a characteristic stromal rich, elevated ROS production and increased drug resistance [23] Pancreatic ductal adenocarcinoma (PDAC) a type of PC is notorious cancer that spread and grow quickly with only $20 \%$ patients suffering from it can be cured reducing the survival rate of patients to less than 5\%. It accounts for $95 \%$ of tumors that are developed in PC. The genetic as well as environmental factor along with smoking habit of individual, diabetes and high fat and cholesterol diet, obesity, and hypertension are known risk factors. High alcohol consumption is also one of other factors for its progression[24]. Cancer arise from inflammation and is critical for its progression. Nearly $20 \%$ of cancers is due to chronic infections. Inflammatory mediators such as growth factors, chemokines, and cytokines release immune cells during inflammation, these in the microenvironment of tumor not only promotes but as well as inhibits inflammation-induced cancer [25]. These inflammatory mediators produce ROS and therefore play a critical role in cancer. $96 \%$ of the pancreatic tumor are of exocrine nature while remaining are neuroendocrine type. PDAC is an exocrine while pancreatic neuroendocrine tumours or PancNETs. [26] There are a number of stress signaling molecules that are involved in the PC and the altered redox hemostasis disrupt its normal functioning resulting either promotion or inhibition of cancer. Some of these are (NRF2), [27] kelch-like protein 19 (KEAP1), NF- $\mathrm{B}$ is a transcription factor and mutation in any of its five dimers can assist PC [28] MYC-C is a targeted oncogene [29].This gene in PDAC is activated by PIN1 along with NRF2 utilizing Kras/ERK/NRF2 pathways which maintains the redox making PIN1 a good target which will restore the redox 
balance. [30] The restoration of signaling molecule mTOR pathway [31, 32] Inactivating mTOR mutations or by synthesizing potential inhibitors of its pathway reduce oxidative stress [31].

Pancreatic cancer originates from pancreatic lesion that are fluid filled sac normally present on or in pancreas. The cancer can be found in epithelium, known as pancreatic intraepithelial neoplasia (PanIN 1-3) or can be fully invasive and the most commonly occurred Invasive ductal carcinoma. In PC a number of growth factors (GF) are overexpressed and its receptors such as EGF and its ligands such as TGF-alpha, GFs and their receptors such as fibroblast growth factors, nerve growth factor, platelet-derived growth factors, and insulin-like growth factors and their respective receptors are overexpressed in PC and contribute to genetic variation and variable phenotype along with ability to rapidly divide and grow. Alteration in growth inhibitory and apoptotic pathways with a significant number of growth promoting factors give PC cells a lead to grow which clinically results in rapid tumor progression and poor survival prognosis. [33] Gastrin is a hormone that is expressed in fetal pancreas where it plays important functions, however it stops its expression at week 14 of embryo. It is not found to be expressed in adults. CCK receptor is reactivated by gastrin during PIN1 in PC which induces signaling through the AKT pathway resulting in cell proliferation [34]. An expression transmembrane receptor insulin-like growth factor-1 receptor ( IGF-1) regulated by interfering RNA (siRNAs) can be seen in PC. vascular endothelial growth factor receptor (VEGFR) are the most commonly used receptors for the treatment of cancer evaluating angiogenesis in PC [35] with its overexpression became a probable biomarker for prognosis [36] and can be used for early detection of PDAC [37] The integrin $\alpha 5 \beta 1$ has a suggested a role in cancer development and progression, the inhibition of this upregulated receptor significantly reduces PC invasion induced by radiation [38] somatostatin receptors (SSTRs) are of 5 types that includes $\underline{\mathrm{SST}}_{1}, \mathrm{SST} 2, \mathrm{SST} 3, \mathrm{SST} 4, \mathrm{SST} 5$. The type 1, 3 and 5 display a major role in inhibiting pancreatic cancer growth.[39] The neuroendocrine type tumor of pancreas express this receptor and targeting this receptor in gastro-entero-pancreatic neuroendocrine tumors has proved to decrease the aggressiveness of the disease[40] cell surface Death Receptors (DR5),the TRAIL bond to DR5 and induces extrinsic apoptosis [41] There has been various reports suggesting the overexpression of claudin-4 receptor to be associated with PC with its expression being reduced with Clostridium perfringens enterotoxin (CPE) and can a good target for treatment,[42-44] the RON receptor and the angiotensin II receptor AT-1 are expressed in pancreatic tumor cells [45],estrogen receptor (ER) [46],cell surface receptor CD44s[47]. transferrin receptor is highly 
expressed in PC[48] with its suggested role to be involved in supporting mitochondrial respiration and generation of ROS thus Significant for PC growth and survival [49]. Targeting the overexpression of Adrenomedullin receptor[50], MUC1 receptor [51] has also been fruitful. The epidermal growth factor receptor (EGFR) belong to family ErbB-1; HER1 in humans is a receptor protein that when binds through one of its ligand provide instruction for cell differentiation and profileration [52]. PDAC is dependent of EGFR signaling [53] . Its uregulation in PDAC increase cell multiplication, promote its growth, progression and survival along with decreased in rate of programmed cell death. EFGR are activated by growth factors as EGF and TGF- $\alpha$ which subsequently dimerize and activates intracellular pathways by autophosphorylating their tyrosine residues. These pathways include Ras/Raf/MAPK, PI3K/AKT and JAK/STAT pathways. Members of cytokines family like IL-1 and TNF $\alpha$ activates NFkB pathway and its activation is observed in PDAC whose inhibition leads to induction of apoptosis in PDAC and increases chemo sensitivity. phosphatidylinositol 3-kinase/AKT, c-Myc, and the Ras/Raf pathway are also found to be over activated in many PDAC cases [54]There has been extensive literature available on targeting these receptors and using it as a potential treatment option for PC. Below in the Figure I are few of the more receptors that are found to be expressed in PC Targeting these signaling protein or its pathways and regulating the expression of the proteins have been exploited by pharmaceutical companies[55] P53 is another protein that is overexpressed in PC and its downregulation decreases pancreatic tumor growth[56, 57]. Another $\mathrm{TF}$ known as GLI transcription factors it was found that GLI-SOX2 signaling axis has a role in PC and the reduced SOX2 expression or down-regulation of $G L I$ transcription factors may be effective in sensitizing PC to gemcitabine treatment.[58] There are significant number of genes that has so far been identified to be mutated in PC and expression is regulated in practice and clinical trial. 


\section{Frequent mutations in Genes of Pancreatic cancer}

Six genes of FPC families' are associated with high risk when it comes to PC mutations and these genes are BRCA1, BRCA2, CDKN2A, TP53 and Lynch syndrome genes such as_MLH1 and ATM .They have been recognized in 5.5 percent of all Pancreatic patients, together with 5.2 percent of disease patients without a family ancestry of pancreatic disease.[59, 60] P53 is among one the most common mutations observed in PC and this mutation results in loss of functioning which may damage the DNA within PC cells and the sensitivity to GME may be due to this mutation. Recent evidences suggest that the increased chemoresistance during the treatment is due to epithelial to mesenchymal transition (EMT) . There are various reports suggesting the use of miRNAs as a regulator of EMT, with miR-200b elevation that can act as potential biomarker for chemosensitivity.[61]RUNX2 is expressed at increased levels in patients suffering from PC and knockdown of this using siRNA has shown to increase sensitivity of GM treatment on PC regardless of P53 mutation [62]. Targeting EMT-TF Zeb1 has also been recommended for regulating EMT [63] Besides these genes there are some mutations like in CFTR that significantly increase the risk of PC[64] BRCA2 genes increases the female chances of acquiring inherited breast or ovarian cancers while elevating man's chance of developing prostate or breast cancers. They also increase the risk of PC and melanoma in both genders. CDKN2A gene is a germline gene, which responsible for carrying information for inhibition of cyclin-dependent kinase 4 [INK4A] and testing is usually recommended for patients with those in which the acquired genes of PC runs in the family. Other mutations that are observed in PC are PALB2, a localizer and partner of BRAC2, STK11, PRSS1, activation of K-ras onco gene and inactivation of TP53. [65] Besides the above mentioned genes, some other genes of FPC families that are also found to be mutated in PC are PALB2, and genes that some other genes that are linked to Lynch syndrome such as MSH2, MSH6, PMS2, and EPCAM. These germline mutations increase the risk of PC as well as other types of cancers. Activation of Kras and inactivation of TP53 Cdkn2A/Ink4A results in the development of PC in mouse.

Recently increasing efforts has been made to cure PC as it's unlike other cancer types respond poorly to chemotherapy specifically CSCs are not eradicated by these treatment which hence make it non curable. A number of serum biomarkers are present for the identification of PC. carbohydrate antigen 19-9 (CA 19-9) is one of the biomarkers that has proved to be involved in PC prognosis however its 
non-specificity to PC and in even patients suffering from PC may not even show level of CA 19-9. [66] The serum level of soluble urokinase plasminogen activator receptor is another receptor which has also been recognized as a probable biomarker for PC patients which are undergoing tumor resection[67]. Detection of CXCR4 may acts as an another uselful biomarker for prognosis of the diseases[68]. KRAS, TP53, CDKN2A ,SMAD4 and P16 genes are the most common mutated genes [69] The diagram II represent an extensive list of mutated genes

Mutations of oncogenes with inactivation of tumor suppressor genes and overexpression of growth factors are involved in PDAC. It is essential to have a better understanding of these pathway that are utilized to synthesize therapeutic drugs that target these proteins and pathways of cancer. Some of the pathways of pancreatic cancer are discussed below

Figure I Pancreatic genes mutations
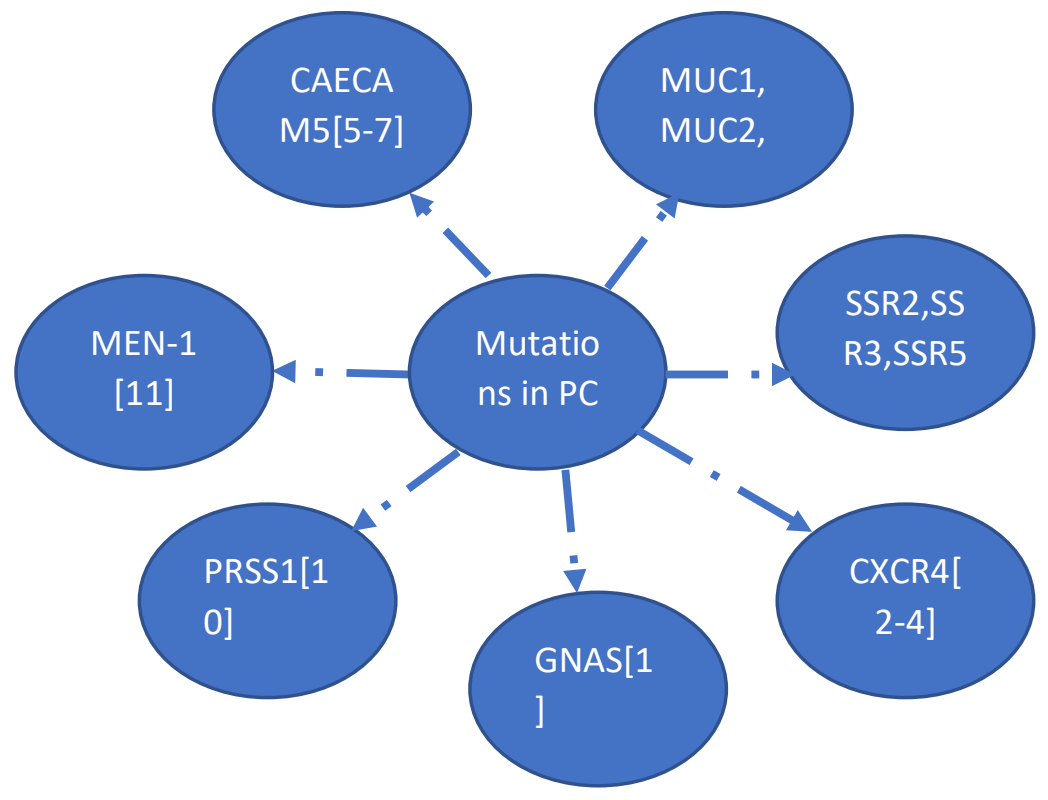


\section{Pancreatic cancer pathway}

\subsection{Apoptosis}

Apoptosis is a programmed or intrinsic cell death which maintains tissue homeostasis through utilizing its pathways. Reprogramming the signaling pathway and acquiring innate resistance to the chemotherapeutic drug can favor in progression or formation of tumor. This is experience in PC[70]. Chemo or radiation therapy works on apoptosis and the alteration to the apoptotic pathway result in the developed resistance experienced during treatment. There are two pathways that are involved in apoptosis i.e Intrinsic mitochondrial pathway and Extrinsic death receptor pathway. Both intrinsic and extrinsic pathways lead to killing of cell. Caspases are cleaved and activated from its inactive form to its active form in response to death receptors, which will then cleave a wide range of substratses which will eventually results in apoptosis. [71, 72] The effector caspases are the main executioner of apoptosis. Cancer cells figure out how to avoid every one of them in the process of their oncogenic transformation by procuring resistance mechanisms. [73]

Apoptosis through ER stress dependent pathway is also reported. The ER stress disturb protein

folding in the ER and generate a cellular response to stumli. unfolded protein response (UPR) is a process that restores this unfolding to balance the hemostasis. However if due to any circumstances the balance cannot be restored the disturbance of cellular processes along with apoptosis occur. ER stress-induced cellular dysfunction and cell death as major contributors to many diseases including cancer[74]

\subsubsection{Intrinsic Pathway/Mitochondrial Pathway/Apoptosome Mediated Apoptosis}

It originate from inside the cytosol of cell and it mainly targets mitochondria. The process is described in the diagram $[75,76]$ 


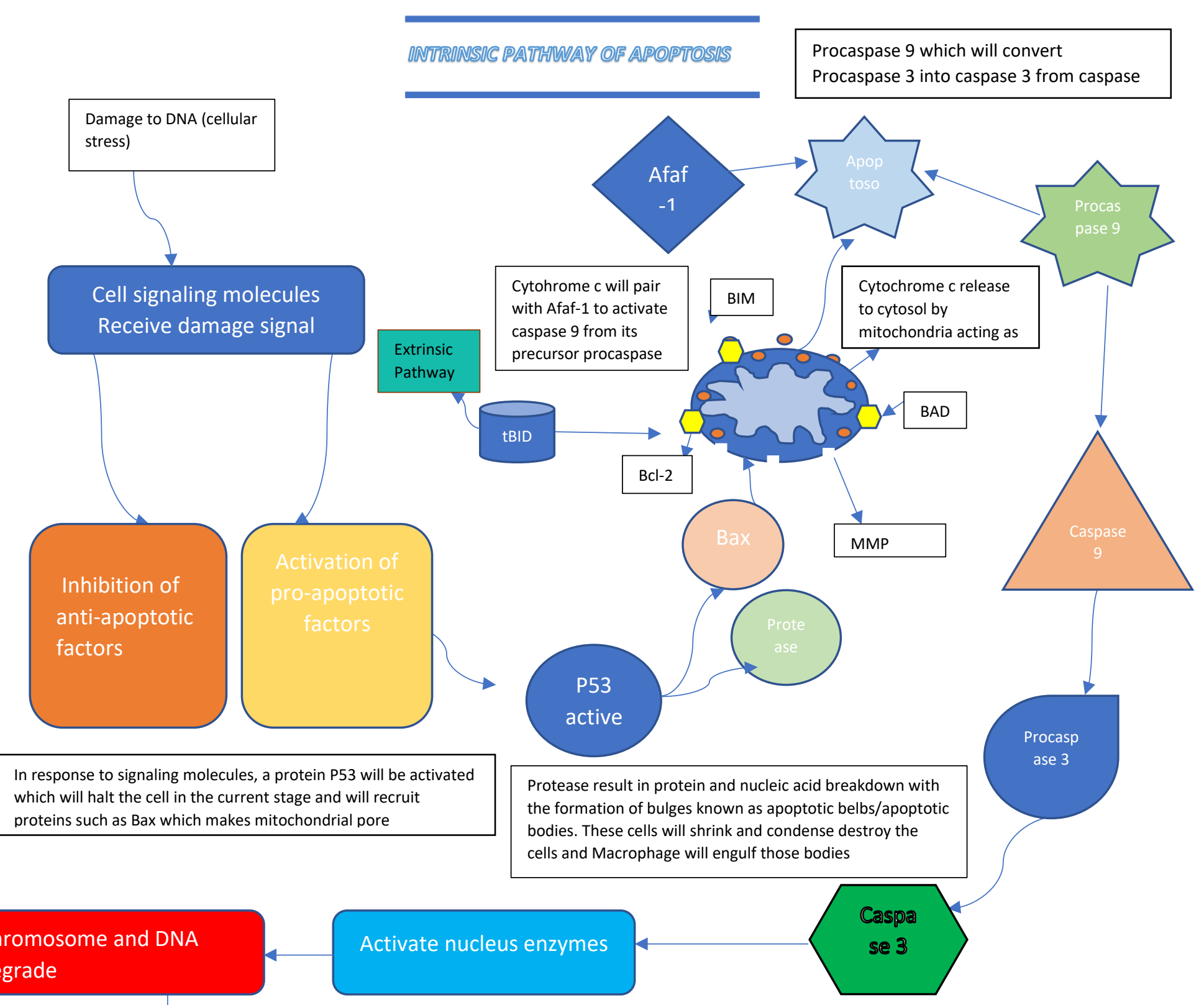

this active nucleus enzymes can go inside and find chromosome and start degrading them and with this degraded DNA, cell cannot survive and hence cell undergo apoptosis via intrinsic pathway
Caspace 3 will activate nucleus enzymes and this does so by degrading the inhibiting enzyme of 


\subsubsection{Extrinsic pathway}

In this pathway the Signal originate from outside the cell. The process is described in the diagram.[77]

\section{EXTRUNSIC PATUWAY OF APOPTOSIS}

Death domain containing receptors are fast receptors. The signal when received from outside the cell due to infection or DNA damage attach to the fast ligand receptor and activate intracellular death domain receptors. Due to multiple receptors of close proximity death receptors will be activated and give death

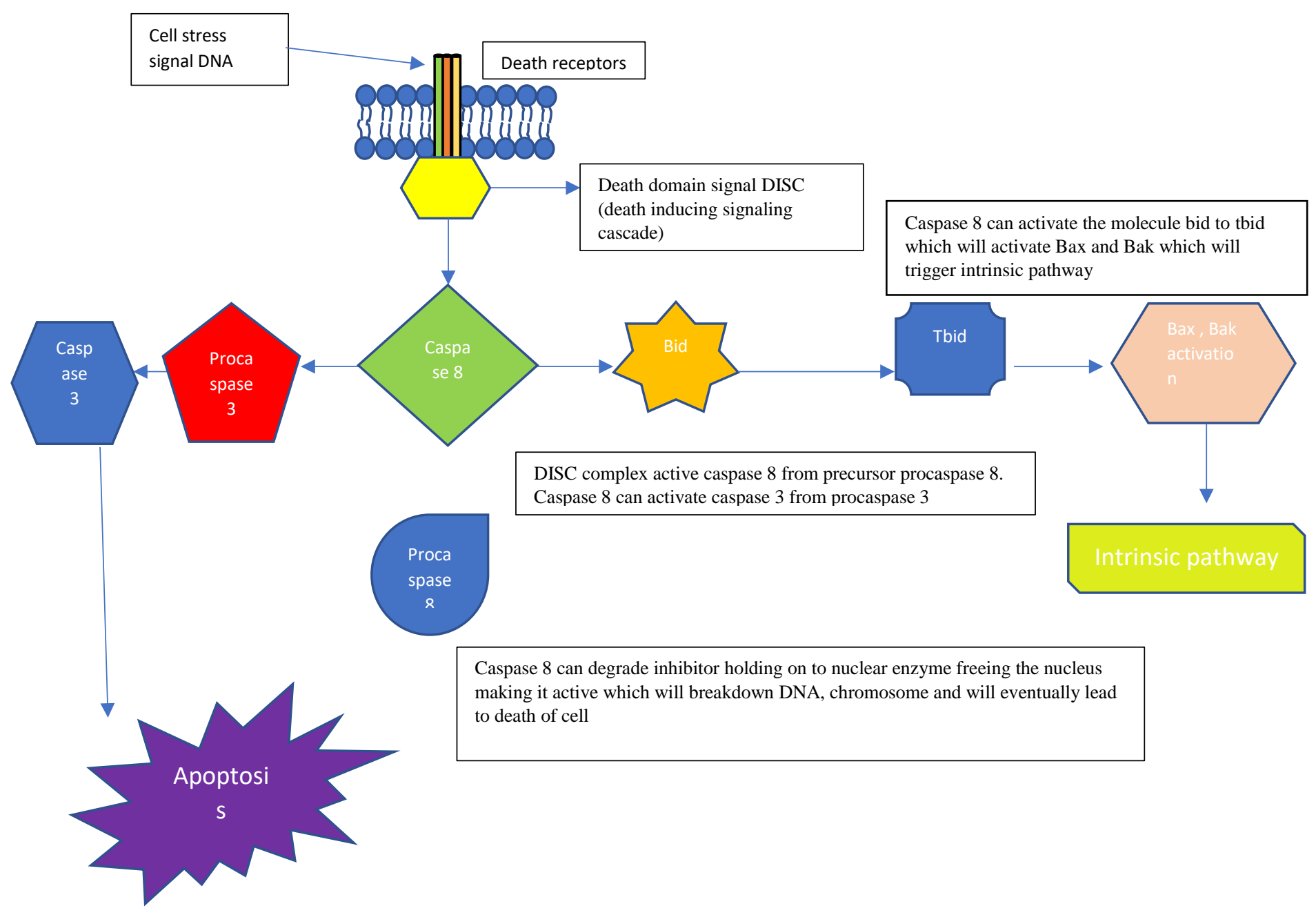

Figure III Extrinsic pathway of Apoptosis 


\subsection{Sonic Hedgehog signaling}

Sonic Hedgehog signaling (Shh) is activated in PC and plays a significant part in embryonic development and CSCs regulation. The expression of Hh ligand is observed in major number of PC cell lines. In PDAC an oncogenic Kras promotes Shh expression via NF- $k$ B signaling. However, role of Hh signaling for PC development, progression and metastasis is not well studied. Using smoothened signals inhibitors for the clinical treatment of PC has failed due to lack of understanding of $\mathrm{Hh}$ signaling [78].NF- $\mathrm{kB}$ expression, MMP9 expression, and Gli1 expression may help in prognosis of PC hence can be utilized as a biomarkers to guide therapies for PDAC in the future.[79] Triacetyl resveratrol upregulates miRNA-200 and suppresses the Shh pathway in PC and was found to be effective in its treatment. [80]

\subsection{Small GTPAs/small G-proteins pathway in pancreatic cancer}

Cancer develops due to mutations in small GTPAs pathways and signaling networks. Rac2, RhoA and Cdc42 are three members of Rho GTPas family. Activation of Rac2, RhoA has effect on fibroblasts and tumor formation. Changes to the Rho GTPase pathways observed in PC along with overexpression of Rac1 GEF( guanine nucleotide exchange factor) such as Vav1. Some reports suggest the blocking of Rac1 binding to its effector PAK1 Following dosedependent activation of EGF induced by Rac1 results in decrease in cell multiplication and migration was experienced in a number of PC cell lines. The animal model of PC using mouse showed the inhibition of GGTase I which was found to be linked with reduced tumor growth in association with the inhibition of protein geranyl-geranylation. GGTase I is in clinical trial however the convincing results has yet to published [81].

Four K-Ras effectors that are characterized by Ras domain has a role in initiation and progression of PC tumor and these effectors are namely; mitogen-activated protein kinase (MAPK) pathway, phosphoinositide 3-kinase (PI3K) pathway, Ral guanine nucleotide dissociation stimulator (RalGDS) and Ras-related C3 botulinum toxin substrate 1 (Rac1) GTPase. These receptors are inhibited usually through MAPK and PI3K pathways. K-Ras effector, the Rac1 signaling pathway is important for $\mathrm{PC}$ development because of its role in the PC initiation and progression. The drugs targeting Rac1 and Rac1/PAK inhibitors of the pathway was found not to effective in animal models therefore the second generation inhibitors of the Rac1 inhibitors that are now recommended are Ehop-016 which blocks Rac1 and Rac3 and AZA1 that blocks Rac1 and Cdc42 and PAK kinase inhibitor FRAX597 that blocks group I PAK kinases. These drugs have been 
proved to be beneficial against mouse models of various types of cancer. However its effect in preclinical trial has yet to determine for making it a PC drug [82]. Kras which encode small GTPases. its activation been identified in more than $90 \%$ of PC. The activation from its non-active to active state is governed by guanine nucleotide exchange factors (GEFs). KRAS inactivation is mediated by GTPase-activating proteins (GAPs), which induce hydrolysis of GTP[83]

\subsection{Transformating growth factor Beta TGF}

TGF $\beta$ signaling has TGA $\beta$ ligand and receptor TGFR $\beta$. The cellular signaling begins after binding of this ligand to receptor. TGF $\beta$ has a number of cellular activators works and through two different pathways that are associated with cell growth, proliferation and differentiation. These allow the cell to gain properties like metastasis and angiogenesis and also imparts functions to convert a normal cell into a cancer cell. This pathway has been targeted for cancer therapy [84]. Galunisertib is a receptor blocker of TGF $\beta$. It was administered to patients with unresectable PC tumor along with chemodrug gemcitabine. The synergic treatment of the two resulted in increase of the survival rate of patients[85] In cancer cells, EMT is controlled by Snail1, a transcriptional factor also required for the activation of cancer-associated fibroblasts (CAF). USP27X is a protein coding gene increases Snail1 stability moreover its downregulation of USP27X decreased Snail1 protein in several tumor cell lines. Depletion of USP27X prevented TGF $\beta$-induced EMT and fibroblast activation. [86] TGF $\beta$ and PD-L1 pathway inhibitors may synergize in PDAC [87].

One another pathway that is involved in PC is the Notch pathway. Targeting Notch pathway with its downregulation contributes to the inhibition and apoptosis of PC cells [88]. Wnt Signaling pathway is also found to be reactivated in various cancers including PC. [89]. chemokine receptor 4 (CXCR4) can modulate this pathway and can acts as drug target [90].The TRIM29 gene is overexpressed in PC with correlation being found to be associated with high level of $\beta$ catenin and the gene activates Wtn and its cascading pathways [91].c-Jun N-terminal kinase signaling pathway,[92] $\beta 1$-Integrin signaling[93] and KRAS signaling [83, 94] are also some of other the pathways that are associated with PC.

\section{Therapeutic approaches to treat pancreatic cancer}




\subsection{Apoptosis}

Colony formation assay determined the anticancer potential of extract of Cordyceps sinensis against human PC cell line MIAPaCa-2 and Capan-1 cells. It inhibited the number of cells that were viable in the cultural medium, its ability to multiplicate and form colony. It also arrested the cell cycle and brought about the early apoptosis of PC cell lines used in a dose dependent and time-dependent manner. The same effect was also observed in vivo. Decrease of $\Delta \Psi \mathrm{m}$ and upregulation of Bax, cleaved caspase-3, cleaved caspase-9, and cleaved PARP as well as downregulation of Bcl-2 both in vitro and in vivo indicated that the mitochondria-mediated intrinsic pathway was involved in cordycepin's antitumor effect [95].

Galectin-9 (Gal-9) is a tandem-repeat type galectin has been studied to evaluate its effect on proliferation of human PC cells and the association of microRNAs and Gal-9 with antitumor effects. In PC cell line Gal-9 decreased cancer cell proliferation, elevated caspase-cleaved keratin 18 level and the expression of cytochrome $\mathrm{c}$ along with reduced the expression levels of phosphorylated EGFR and various receptor tyrosine kinases (RTKs). Hence it induced intrinsic apoptosis in PC cell lines through the caspase-dependent and caspase-independent pathways. Gal-9 suppressed tumor growth and may acts as a new pharmaceutical target for the PC treatment.[96]

Capsaicin dose-dependently induced G0/G1 cell cycle arrest and apoptosis. The knockdown techniques was used to reduce the expression of DNA damage-inducible gene 153 (GADD153) which is a marker of the ERS mediated apoptosis pathway.[97].The knock down was carried by siRNA which reduced the capsaicin-induced apoptosis in both PANC-1 and SW1990 cells. The in vivo studies using xenograft tumor-induced mice also confirmed the capsaicin ability to significantly inhibit the growth and metabolism of PC along with increasing the survival time. In addition capsaicin also increase the expression of some key ERS markers, including glucose-regulated protein 78 (GRP78), phosphoprotein kinase-like endoplasmic reticulum kinase (phosphoPERK), and phosphoeukaryotic initiation factor-2 $\alpha$ (phospho-eIF2 $\alpha$ ), activating transcription factor 4 (ATF4) and GADD153 in tumor tissues.[98]. Chemotheraphy promotes oxidative stress which activates Nrf2 and produce ROS which induce autophagy. Nrf2 regulates ARE driven gene expression and its overexpression of Nrf2 has been observed in PC. Bortezomib induce PC cell apoptosis and ER stress. It also increases ROS which activates Nrf2 and autophagy. Bortezomib (BZ) is a highly selective proteasome inhibitor, with chemotherapeutic 
potential however due to chemo sensitization, its effect alone is not sufficient and combining it with drug that can co inhibit autophagy and Nrf2 signaling could be a promising combination therapeutic approach for PC treatment.[99]. When a PCC cell lines were treated with BZ, it demonstrated a defective phosphorylation and delayed translational arrest of Eukaryotic Initiation Factor 2 (eIF2). As a result ubiquitylated protein aggregates and ROS was produced which induced apoptosis. The phosphorylation may be attributed to stress, a serine kinase target, in response to the presence of Heme deficiency (HRI). It was found that HRI was responsible for this resistant cells and were abundant in BZ activated eIF2 $\alpha$ kinase. These findings may suggest an alternate pathway for therapeutic agents.[100]

Activation of signal transducer and activator of transcription 3 (STAT3) is usually observed in different cancer types including PC and therefore are mostly targeted for treatment of cancer. The activation is followed by cellular transformation and tumor formation. HO-3867 acts as an inhibitor of STAT3, ROS-dependent ER stress pathway and induce cancer cell apoptosis thus can be targeted for therapy of PC.[101]

Various physiological functions including glucose and oxygen insufficiencies, chemotherapy agents induce ER stress which activates UPR signaling which help recover the cells from stress. GRP78 is a regulator of the UPR and has found to be upregulated in PC with a role in multiplication, promotion and invasion tumor. In PC CD133+ or AC133 acts as CSC biomarker which shows a role being associated with cell self-renewal, tumorigenesis, metastasis, resistance, metabolism, differentiation, autophagy, apoptosis, and regeneration [102]. The study shows that an overexpressed GRP7827 have a significant role in the survival of normal stem cells however its association with CSC has yet to be determined. UPR signaling keep the ROS level at minimum and transcriptionally regulate the enzymes that are involved in the mechanism of detoxification. The cancer cells have diverse surrounding microenvironment with an ability to react to hypoxia and hyponutirent condition. This enable them to change their bioenergetics by a process of metabolic reprogramming. [103, 104] The CSC also undergo this phenomenon to lower ROS level which may cause genetic material destruction and instability of genomic. Through Metabolic reprogramming of CSCs it maintains ROS level to minimum which prevents the DNA damage and genetic instability. Lipid hemostasis along with oxidative stress is also essential for the normal and cancer cells. When cancer cells are multiplying cells depends on high level of cholesterol and lipid which is provided by either upregulation of 
lipogenesis pathways or from exogenous source. Therefore, disruption of ER stress regulation affects these processes as well. PC cell line used were stably expressing shGRP78 was used to determine ER stress regulator and its effect on phenotype of PC. The pathway that responsible for proliferation survival, fatty acid metabolism, and cell organization and biogenesis are affected when GRP78 is downregulated. It also maintains the balance of ROS species and the reoccurrence characteristics of PC. The elevated UPR in PC give cells its "stemness" properties which are associated with aggressive properties like chemoresistance and metastasis.[105]

\subsection{Inhibition of PI3K/Akt pathway}

\section{Inhibition of PI3K/Akt pathway through Polo-like kinase 1 (PIk1) :}

The observed activation PI3K/Akt pathway and efforts for its inhibition resulted fruitfully in induced apoptosis of various cancers. Plk1 functions in controlling apoptosis and mitosis with its overexpression being observed in various cancer cells. The report suggested the induction of apoptosis with decreases of cell multiplication by downregulating Plk1 can achieved by inhibition of PI3K/Akt pathway. Furthermore, Plk1 inhibition results in programmed cell death of cancer cells by inactivating XIAP, activating caspase-3, upregulating BAX and downregulating Bcl-2. Using the information and the associated molecular mechanism of PI3K/Akt pathway and Plk1 in that are involved in PC cell proliferation and apoptosis may provide a novel approach for chemotherapeutic agent [106].

\subsection{Inactivation of JNK pathway}

Hyperglycemia can affect pancreatic tumor growth, progression, invasion and migration, proliferation by elevating ROS and inducing oxidative stress. Activation of the JNK pathway is done by ROS species, its inactivation can be a useful therapeutic approach.[107] Targeting it in combination with FU- or GEM-based regimens may be a useful therapeutic approach to overcome resistance of PCCs.[108]

\subsection{Activation of ERK/ EFRG and JNK pathway}

\section{Activation of EFRG/ERK pathway}


Association of glycometabolism with progression of PC has been recently identified. HIF-1aplha is an important factor in glycometabolism of cancer. It functions by regulating tumorigenicity through GLUT1.It regulates oxidative stress under inadequate oxygen supply in PC. Controlling ROS increase EFGR which effects glycometabolism of cancer using ERK1/2 pathway. Activation of EFRG/ERK pathway mediated by HIF-1aplha regulate glycometabolism of PC[109]

\section{Activation of ERK and JNK}

Sophoridine is a quinolizidine alkaloid isolated from traditional Chinese herbs having antitumour potential by inducing ROS mediated apoptosis. The mechanism involves the inhibition of ubiquitin-proteasome pathway which arrest cell cycle at S phage of PCCs, and induces apoptosis using inhibitors of MAPK signaling pathway. Sophoridine has the ability to stop the PC cell line growth in vitro and reduce the volume of xenograft tumors in vivo. The dose dependent amount has been proved as preclinical drug with ability to decrease volume and mass of tumor as compare to control group. Two ROS dependent pathways ERK and JNK were found to be activated in compound treated tumor tissues with less proliferation in cancer cells, and more apoptotic cells in Sophoridine-treated tumors.[110]

\subsection{Activation of JNK}

\section{Capsaicin-treatment resulted in the activation of JNK and JNK inhibitor}

Capsaicin is a major pungent ingredient of red-chili pepper. It has the ability to induce apoptosis , cell viability, activation of JNK and increase the production of ROS with constant disturbance of MMP, release of cytochrome $\mathrm{c}$ in the cytosol and activation of caspase- 3 cascade in PC cell lines AsPC-1 and BxPC-3 cells. Capsaicin treatment with these cell lines resulted in increased expression of Bax, down-regulation of bcl-2, survivin and significant release of cytochrome $\mathrm{c}$ and AIF in the cytosol. It activated JNK and JNK inhibitor SP600125 afforded protection against capsaicin-induced apoptosis. The capsaicin is given orally to the athymic nude mice resulted in reduction of tumor growth of AsPC-1 by increasing the apoptosis and activation of JNK along with an increased cytosolic protein expression of Bax, cytochrome c, AIF and cleaved caspase-3, as compared with controls. Hence the capsaicin effectively acted as an efficient anticancer agent both in vivo and in vitro. The proliferation was of the cell lines was inhibited without affecting the 
viability of acinar cells derived from normal human pancreas. The in vitro study confirmed the antitumor effects of capsaicin in vivo in AsPC-1 tumor xenograft model.[111]

\subsection{GPx1 silencing utilizing Akt/GSK3ß/Snail signaling}

Glutathione peroxidase-1 (GPx1) modulates ROS by eliminating hydroperoxides. EMT is involved in the chemoresistance in tumors. GPx1 silencing resulted in the EMT and GEM resistance accompanied by activation of Akt/glycogen synthase kinase $3 \beta$ (GSK3 $\beta$ )/ Snail signaling. EMT and chemoresistance is inhibited by GPx1 by using Akt/GSK3 $\beta /$ Snail signaling pathways in PDAC. GPx1 acts as a novel biomarker for predicting the response to GEM-based chemotherapy.[112]

\subsection{Activating NF-кB/STAT3 pathway}

STAT3 signaling is associated with chemoresistance and is a molecular target in PC therapy. Its role in response to gemcitabine treatment and subsequent chemoresistance of PC was studied. Activation of STAT3 might promote CSCs on gemcitabine treatment in PC. Gemcitabine produces ROS which assist the resistance by activating antioxidant response $\mathrm{NF}-\kappa \mathrm{B}$ which is controlled by ROS and has role in malignant behaviors of tumor which activates CSC and aid its survival, growth and multiplication. The treatment with Gemcitabine supported the CSC phenotype in PCCs by activating the NF- $\mathrm{B}$ /STAT3signaling cascade through Nox-mediated production of ROS. Inhibiting the pathway in synergic treatment with gemcitabine can effectively increase the potency of chemotherapy by suppressing pancreatic CSCs [113].

\subsection{Activating SHH pathway}

There are a number of drugs available for treatment of PC, the first line medication is Gemcitabine. Its efficiency is limited due to natural resistance of PC cells. Tumor hypoxia activates TF such as (HIF-1alpha) along with upregulation of heme oxygenase-1 (HO-1) molecules which supports PC cells during oxidative stress. This upregulation along with several other factors are also activated in response to stress and generation of ROS species which protects the body during inflammation associated complications. This upregulation of HO-1 is found in PC cells with its inhibition being associated with increased sensitivity during gemcitabine treatment. Therefore, one method for treatment of PDAC is targeting HO-1 molecule. It was found that using both in vitro and in vivo techniques in the presence of HO-1 inhibitors such as zinc protoporphyrin, tin protoporphyrin IX 
(SnPP) under insufficient oxygen supply, PCCs become more tumour resistant to gemcitabine treatment. Treating the tumor with SnPP alone and in combination with Gemcitabine resulted in the reduction of tumor weight, decreased metastasis and increased the efficiency of the used chemo drug when used in combination. The Caspase 3 activity was found to be detected high when treated with the synergic treatment .Therefore the use of HO-1 inhibition and gemcitabine under insufficient oxygen supply can be beneficial as it can suppress an in vitro multiplication of PC cells but also cancerous growth in vivo..[114] One other report suggesting the overexpression of HO-1 is related to increased sensitivity and proliferation and that the use of HO-1 inhibitor on PC cell line MIA PaCa-2 and PANC-1 decreased the cell proliferative ability by activating SHH signaling pathway. [115]

\subsection{Reduction of gene expression}

\subsubsection{Reduction of gene expression of sirtuin (SIRT)4 using chromatin modifier UHRF1}

Patients suffering from PC has low survival rate, with resistant to chemotherapy and radiation treatment. Epigenetic modification of tumor suppressors occur in PC which causes mutation in these suppressors along with its silencing by the addition of methyl to a DNA molecule, a process known as DNA methylation and chromatin modifications, which can be by modifiying chromatin contacts or by making changes to nonhistone proteins to chromatin in the promoter region. A chromatin modifier UHRF1 is found to be upregulated in different types of cancer cells and plays an important role in progression and oncogenesis of PC cell. Oncogenic mutations in PC reprogram cancer cell metabolism. UHRF1 increased PC cell growth by the use of oxygen and transforming glucose into lactate by the process of aerobic glycolysis and decreased hypoxia inducible factor (HIF1) $\alpha$ protein levels. UHRF1 reduces the gene expression of sirtuin (SIRT)4 which is located in the mitochondira and is a negative regulator of mitochondria of aerobic glycolysis and an antioncogene and play a role in oncogenic stress. $[116,117]$

\subsubsection{Targeting dCK expression}

5-fluorouracil (5-FU) and Gemcitabine are the most commonly used pancreatic cancer drugs. The latter being a proto drug has to be transformed into its active form which takes place intracellularly by deoxycytidine kinase (dCK) by phosphorylating it to mononucleotide active drug. $\mathrm{dCK}$ functions as a catalyst in the process by catalysing the rate- limiting step in gemcitabine metabolism. Its impact on 
pancreatic cancer proliferation was reported. It suggested that the decreased expression of dCK results in NRF2/ARE hyperactivation associated with increase in the level of ROS reinforcing gemcitabine resistance. This signaling pathway decreased expression

of ARE- driven antioxidant genes. dCK exhibit tumor suppressive role PC cell lines.[118]

\subsection{Nrf2 Regulation}

Nrf2 also known as Nfe212 and its suppressor protein Keap1 is seen overexpressed in PC. Changing the activity and level of Nrf2 may display a potential role reducing the PC growth and increase its sensitivity to the developed drugs.[119] The deregulation of different pathways such as $\mathrm{PI} 3 \mathrm{~K} / \mathrm{AKT} / \mathrm{mTOR}$ is observed in tumor cells and is thought to promote cell survival through Nrf2 activation. [120] PC cells have the inborn ability to show resistance against chemotherapy drugs and this resistance is due to abnormally elevated Nrf2 protein levels. This thus opens a possibility of developing drug that results in regulation of Nrf2 protein levels. Increased drug resistance is observed in PC with increased protein level of Nrf2 which can be due to overexpression of exogenous Nrf2 or by activating endogenous Nrf2. Drug changes its resistance with expression levels of Nrf2 downstream genes such as ABCG2. [121]

\subsubsection{TGF- $\beta 1$ and Nrf2}

TGF- $\beta 1$ is a secreting protein belong to cytokinin and plays role in both tumor suppressor and promoter depending upon stage of tumor and cellular context [122] making it a predictive biomarker for treatment of cancer[123, 124]. Dysregulation of its signaling pathway is associated with various cancer types [125]. Clinical trial have been conducted to target TGF- $\beta$ signaling for treatment of PC [126] TGF $\beta$ binds to its receptor forming TGFBR whose inhibition has been proved to be effective in PC treatment especially in patients who has already lost tumor suppressive TGF $\beta$ signals in the epithelium[127]. MUC1 protein which is also a of TGF- $\beta$ inhibitors may regulate TGF- $\beta$ function in PDA cells and thus have potential clinical trial [128] .

\subsection{2 inhibiting Nrf2 signaling}

Metabolic stress induces epigenetic alteration and overexpression of Nrf2. This upregulation causes the cancer cells to protect itself from apoptosis and adapts enhanced proliferation favoring the tumor formation as well as resistance to chemo and radiotherapy. Nrf2 protects the cells from oxidative stress and maintain cellular redox hemostasis. Its activity is suppressed by the formation 
of complex when Keap1 connects Nrf2 and Cul3 dependent E3 ubiquitin ligase. Upon the disruption of normal redox hemostasis, Keap1-mediated proteasomal degradation is inhibited by ROS causing the translocation of Nrf2 to the nucleus. Then Nrf2 binds to AREs and enhances transcription of its target genes. Its overexpression is associated with the developed drug resistance experienced during chemotherapy. The use of Nrf2siRNA to knockdown Nrf2 can be effective against this chemo resistance. Tamoxifen is also reported to have positive impact on chemo resistance. Drug resistance increased with overexpression of $\mathrm{Nrf} 2$ and decreases with its downregulation in PC. This makes it a target to reverse chemo resistance experienced during treatment. Digoxin is a cardiac glycoside used in heart failure with recent reports suggesting its use in cancer therapy by inhibition of proliferation, induction of apoptosis. Digoxin has the ability to inhibit activity of the Nrf2-ARE luciferase reporter gene in A549-ARE cells making it a potential Nrf2 inhibitor through suppressing phosphate dylinositol-3 kinase (PI3K)/Akt signaling pathway. It also reverse drug resistance of gemcitabine in PC cells. Thus, digoxin might be a promising agent to reverse gemcitabine resistance in gemcitabine-resistant pancreatic cancer cells via inhibiting Nrf2 signaling.[129]. Natural compounds such as coffee alkaloid trigonelline (trig) inhibit Nrf2 with a decreased proteasome activity, leading to an elevated sensitivity to apoptosis. [130]

\subsubsection{Inhibiting Nrf2-Keap1protein-protein interaction}

ROS promote cancer is regulated by the transcription factor Nrf2 and its repressor protein Keap1.Nrf2 is physiologically regulated while neoplasia which is uncontrolled growth of cells that is not under physiologic control is found to be elevated upon basal expression of Nrf2.[131] .Somatic mutations disturb the Nrf2-Keap1 protein-protein interaction (PPI). It is required to steady the function of Nrf2. Increasing the transcription of its targeted genes show elevated ROS detoxification and tumor formation. Endogenous oncogenic alleles of Kras , Braf and Myc suppress ROS. K-Ras(G12D), BRaf(V619E) and Myc(ERT2) each increased the transcription of Nrf2 to stably elevate the basal Nrf2 and lower ROS to establish hemostasis. Using these oncogenes to increase the expression of Nrf2 is novel suggested mechanism for the activation of the Nrf2, and is evident in human PC. Moreover, Targeting oncogenes of Nrf2 pathway impairs K-RasG12D-induced proliferation and tumorigenesis in vivo .Thus, Nrf2 antioxidant and cellular detoxification can acts as mediator of oncogenesis.[132] Currently various efforts has been made to target this Nrf2-Keap1protein-protein interaction for its 
inhibition can be effective in the cancer treatment.[133] However due to safety issues the clinical validity for its activation has yet to be proven[134]. Chlorophyllin is made from chlorophyll has the ability to form molecular bond with other chemical agents which are known to cause cancer. Chlorophyllin sodium cupper salt a derivative of chlorophyll and a semisynthetic mixture inhibits Keap1-Nrf2 protein-protein interactions via activation of Nrf2. Bonaphton a chemo drug also display the same activity. These two along with its analogues has a potential to be of clinical trial with the ability to act through Nrf2 activation. [135] New ligands that can increase oxidative stress response by acting as a competitive ligand for binding of Nrf2 to Keap1. E78P peptide is one suggested ligand which share the same properties and boost oxidative stress. [136]

\subsubsection{Targeting expression of Nrf2}

Humans detect and response to oxidative stress using Nrf2 and Keap1. Keap1 targets Nrf2 for ubiquitylation, leading to its proteasomal degradation. However the dysfunctioning is observed between them under imbalance redox hemostasis with a resulted stabilization and accumulation of Nrf2. Using five different PC cell lines the expression of Keap1 was observed. Nrf2 target genes AKR1c1 and GCLC were detected in PC cell line. Expression of Nrf2 was found to greater in PCCs. Treating the level and expression of Nrf2 therapeutically may reduce pancreatic tumor growth, and increase sensitivity to therapeutics.[119]

\subsubsection{Activating Nrf2 and Decreasing expression NAF-1 using resveratrol}

The outer membrane of mitochondria and its associated membranes along with ER has an autophagy factor known protein nutrient-deprivation autophagy factor1 (NAF-1). The instructions for this protein are provided by CISD2 gene. NAF-1 has association with life span in mammals with mRNA expression level of NAF-1 increased in younger mice than in older mice. Cancer cells adopts to a longer lifespan. The involvement of NAF-1 which antagonizes Beclin-1 -mediated cellular autophagy at the ER was considered. A natural polyphenolic compound, Resveratrol (RSV, trans-3, 5, 4-trihydroxystilbene), both pro and antioxidant potential and can reduce level of ROS by oxidative stress-induced apoptosis or upregulate of ROS causing apoptosis, membrane, DNA disruption and autophagy. It acts against PCCs by decreasing its growth using NAF-1 signaling. Redox-sensitive transcriptional factor Nrf2 is present in cytoplasm in inactive state via tethering to Kelch-like ECH associated protein (Keap1) and gets activated under oxidative state. The TF dissociates with Keap 1 and interact with ARE by moving inside nucleus. Resveratrol 
could activate Nrf2 and suppress the expression of NAF-1 in PCCs by inducing the accumulation of ROS. Decreasing expression NAF-1 hindered PCCs proliferation and activated apoptosis. The targeting of NAF-1 using resveratrol can improve the sensitivity of PCCs to gemcitabine. Thus targeting NAF-1 with the currently used chemotherapeutic agent may enhance the efficacy of therapeutic agent (gemcitabine) in PC therapy.[137]

\subsection{Kras Mutation}

There exists genetic diversity in patient's suffering from PDA. Kras is the most commonly mutated gene found in PDAC. ARID1A mutations in the PDA is also observed where the survival rate of patients having gene mutated is less than those in which it is not mutated thus may acts as prognostic marker for PDA[138] The differentiated state of mature cells or organs has hidden plasticity which allow them to develop in response to environment and deviate functionally from other cell types [139]. Such type of natural endothelium plasticity is also observed in Pancreas which may have effect on tumor progression, translation and initiation. Pancreatic cellular plasticity in neoplastic transformation in response to its environment and its potential role in the generation of CSCs has been investigated. Formation of tumor occur with the mutation of tumor suppressor gene when it is in an undifferentiated cell type. In PDAC mostly the mutation is observed in Kras gene in differentiated cell with the clear compartment of CSC. The process initiated by Kras require repression of epithelial differentiation program and suppression of those factors that are normal activated during pancreas embryonic development. Also the signaling pathway involved during this development restore its activity and contribute to tumor formation. A diverse heterogeneity is also observed in cancer cells itself which has a subset of larger population of its cells that are marked by differential expression of certain marker. Furthermore, fibroblast present in the cellular component of microenvironment also plays a role in both tumor formation and suppression. However, its specific role is poorly understood. Development of better understanding of the composition of surrounding environment of cancer cells will help us develop drugs that are more effective in its treatment. Pancreatic cancer cells with inherit plasticity of transformed cells, late detection of the disease and poor understanding of the basic functions and signaling pathways that are associated with it make it difficult to synthesize a drug that can effectively cure it.[140] 


\subsubsection{Targeting suppressor gene PIN1}

ERK kinase are protein signaling molecules that are involved in the regulation of F-box and its WD Repeat Domain-Containing 7 (FBW7) ubiquitination. FBW7 is a Mitochondrial negative regulator of respiration in PCCs highlighting Kras/ERK/FBW7 axis in promoting PDAC progression. A study was performed to understand the effect of PIN1 gene in PDAC mediated by K-ras on redox balance. A decreased cancer cell growth and apoptosis was observed with downregulation of PIN1 and this may be attributed to mitochondrial dysfunction. Silencing of this gene damaged basal mitochondrial function by elevating intrinsic ROS level. PIN1 sustain the production and formation of ROS by collectively activating c-Myc and $\mathrm{NrF} 2$ to upregulate expression of antioxidant response element (ARE)-driven genes in PDAC cells. Thus, Kras/ERK/NrF2 enhances the growth of tumor and establishes PIN1 as a therapeutic target which can alter the redox hemostasis in PCCs[30].

\subsubsection{Reduction of expression of Nrf2 which decreases Kras generated ROS}

Oncogenic allele of KRAS activation increase ROS which upregulates GF signaling and result in formation and progression of pre precursor lesion that can develop PC cells. Upregulation of antioxidant genes balance the ROS level produced due to KRas-driven neoplasia. The therapeutics involves the drug that can inhibit the expression Nrf2 reduces KRas-generated ROS to target PDAC progression. Alternate line of treatment is the administration of mitochondrially-targeted antioxidants such as mitoQ. Once the cancer is progressed the treatment should be focused on elevating ROS level however for preventation of caner the level should be aimed to decrease.[141]

\subsubsection{Use of RNA interface to target Kras}

The use of nucleic acid sequencing such as siRNA to target Kras has been proposed by various scientists to treat different types of cancer[142] mRNA-17-92 with miRNA genes that collectively encodes 15 mRNAth which are used for development and hemostasis with their deregulation and mutation results in genes [143] can cause cancer of various types and its dysregulation and down or upregulation may result in tumor formation. In most cancer regions an elevated level of miR17-92 cluster can be seen. It targets E2F TF which plays a role in apoptosis and DNA replication. miR-21 increases tumor formation by targeting s PTEN, TM1, and PDCD4, the suppressor genes of tumor. RAS oncogene family constitute three members H-Ras, N-Ras and K-Ras. These are 
converted into its active form by either point mutation or amplification of wild type gene. The active altered form is present in variety of cancer types.[144, 145]

\subsubsection{1 miRNA-96 as tumor suppressor of Kras driven cancer}

miRNA-96 Suppresses the mutationally activated KRAS in PC. Abnormal gene expression of miRNA-96 is done by synthetic miRNA precursor which block KRAS, dampened Akt signaling, and induce apoptosis in cells. miR-96 also reduce the metastasis and spreading of cancer cells to other organs and tissue and decrease the cancer progression in such a way that is related with KRAS downregulation. It acts as an efficient regulator of KRAS with a possible role in curing the PC.[146]. Akt is overexpressed in various cancer types including PC. miR-96 could inhibit the activation by phosphorylation of Akt pathway which is a downstream of KRAS. Xenograft mouse model indicated that miR-96 to a greater extent inhibited the oncogenesis of PC cells. Thus, miRNA is an antioncogene and hence can make a new medicinal approach for curing the PC.[147]

Kras undergoes mutation to form cancer and the most common of which is observed in gain-offunction substitution mutation of glycine at codon 12 to aspartate (G12D). Parenteral controlled drug delivery systems is a method used to control the slow release of drug which is imbedded in biocompatible, biodegradable polymer matrix. For the purpose of experiment, it was named as local drug eluter (LODER) which contained siRNA such as anti-KRAS ${ }^{\mathrm{G} 12 \mathrm{D}}$ siRNA (siG12D LODER). The designed LODER has the ability to release the drug slow over an extended period of time within the tumor as well as protect it from degradation. To determine the silencing potential of siG12D, a specific siRNA directed against the mutant KRAS ${ }^{\mathrm{G} 12 \mathrm{D}}$, Panc1 human pancreatic carcinoma cells that bear this specific mutation in the KRAS gene was used. Local prolonged siRNA delivery system (Local Drug EluteR, LODER) shedding siRNA against the mutated KRAS (siG12D LODER). The stabilized and active LODER-encapsulated siRNA was used where its treatment of PDA cells with siG12D LODER resulted in greater decrease in KRAS levels, leading to inhibition of proliferation and epithelial-mesenchymal transition. In vivo, siG12D LODER prevented the growth of human PC cells and increased the survival of mouse. The siG12D LODER thus have therapeutic potential making the mutated KRAS indeed a drugable target. [148] A clinical trial of the designed drug, siG12D LODER was performed on 15 patients suffering from non-operable stage three LAPC. The drug was delivered to the targeted site containing solid tumor using endoscope ultrasound (EUS) biopsy procedure. During the course of 
LODER implantation, patients also received Gemcitabine chemotherapy 16 days after the LODER treatment. Serum-based tumor marker CA19-9 was found to be decreased in $70 \%$ of patients with synergic treatment and eventually reached a normal level. By the end of nine months CT scan was performed which showed a significant decreased in tumor growth. [149]

\subsubsection{Upregulation of exosome CAT, SOD2 and DCK}

Exosomes are spherical shaped bilayer mammalian Extracellular vesicles (EVs) [150], shed by normal as well as PC cells using glypican-1 surface markers. Conditioned media of PCCs were treated with gemcitabine. Exosomes was found to be the major factor in the chemoresistance by elevating the production of ROS with an upregulation of superoxide dismutase 2 (SOD2) and catalase enzyme (CAT) which acts as a carrier of mRNAs and non-coding miRNA. This upregulation of exosome mediated transfer is likely through lateral transfer of their transcripts and through miR155-mediated downregulation of gemcitabine-metabolizing enzyme, deoxycytidine kinase (DCK) Gem-Exo-treated cells. It was also found that CAT, SOD2 and DCK as important genes involved in exosome-mediated PC chemoresistance. [151] Another study was performed that showed the role of mesenchymal stem cell (BMSC)-derived exosomal microRNA-126-3p (miR-126-3p) inhibited the development of PC through the downregulation of ADAM9, highlighting the potential of miR-126-3p as a novel biomarker for PC treatment. [152] An engineered Exosomes (iExosome) has also been suggested to effectively treat PDAC. It has the ability to deliver iRNA and inhibit tumor formation and progression. CD47 present on exosome increase its half-life while macropinocytosis in Kras-mutant cancer cells favor their exosomes uptake and protects exosome from phagocytosis. iExosome were used to carry short hairpin RNA and target Kras ${ }^{\text {G12D }}$ with increased CD47 dependent efficiency and this process is facilitated by the micropinocytosis mechanism. Treatment with iExosomes suppressed cancer in animal model of PC and to a great extent increased the overall survival. Thus this approach can be used for the direct and specific targeting of oncogenic KRAS in tumors using iExosomes.[153]

\subsubsection{Inhibition of lysosomal acidification}

Survival of Kras dependent PDAC depends on reprogrammed metabolism which among various other factors also includes lysosomal degradation of proteins. Mutation in KRAS increase lysosomal acidification. Macromolecules are transported into lysosome and degraded into amino acid for production of nutrient supply and important biosynthetic reactions. The novel medicinal approach to treat PDAC thus involves the disturbance of lysosomal catabolism by inhibition of 
lysosomal acidification. The use of ultra-pH sensitive nanoparticles can treat PC by releasing the drug rapidly and lysosomal $\mathrm{pH}$ buffering.[154]

\subsubsection{Micropinocytosis inhibition by targeting SDC1}

It is well known that activation of macropinocytosis by KRAS in transformed cells occur through Syndecan 1 SDC1 which serves as a KRAS effector by inducing and regulating macropinocytosis in KRAS driven PDAC. However pharmacological inhibition of macropinocytosis has not been accomplished. SDC1 should be considered as a target for treatment of PDA. Monoclonal antibodies directed against SDC1 (CD138) are being tested for activity in multiple myeloma as antibody-drug conjugates, proving the feasibility of developing targeted therapeutics against this molecule. [155]

\subsubsection{Inhibition of Autophagy and ERK MAPK}

\subsubsection{1 through inhibitor chloroquine}

PDAC is characterized by KRAS mutation and tumor growth and formation that is dependent on autophagy. The efforts to block the Ras protein directly has yet not been successful and the alternate approach of inhibiting it indirectly by blocking the facilitating protein or pathways that are associated with Ras[156]. Suppression of KRAS increase autophagic flux, as well as the inhibition of its effector pathways ERK. It also decreases the metabolic pathway of glycolysis as well as the functioning of mitochondriaPDAC dependence on autophagy and to some extent reduces other KRAS or ERK-driven metabolic processes. By using the inhibitor of autophagy such as chloroquine and by the use of gene inhibition therapy, an inhibitor that is specific autophagy regulators together can increase the efficiency of ERK inhibitors to mediate anticancer activity in KRAS-driven PDAC. Thus the synergic treatment of utilizing therapeutic inhibitors that can simultaneously inhibit both ERK and autophagy processes that are upregulated in response to ERK inhibition may be effective treatments for PDAC.[157]

\subsubsection{2 through inhibitor Hydroxychloroquine}

Another study that shows the dependence of KRAS on autophagy. The study suggested that upon the presence of inhibitors of KRAS pathway, tumor become reliant on autophagy for survival, thus the efforts should be focused on the removal of this dependency by targeting and inhibiting MEK or ERK kinases combined with inhibitors of autophagy will have pharmacological important for 
patients with PDAC. Hydroxychloroquine is autophagy inhibitor is in clinical trials for PDAC.[158]

Changes in the acquired DNA can influence the inflammatory tumor microenvironment and cancer sensitivity. IDO2, is a gene of protein Indoleamine 2,3-dioxygenase 2 has been suggested to be a modifier of immune escape in cancer cells as well as responsible for causing the inflammation associated with it. It is expressed at a higher level in PDAC.[159] Gene deletion of IDO2 has an effect on the PDAC development and its response to therapeutic treatment.. IDO2 genotype analysis can be beneficial for decision making process of further treatment especially for those who can benefit from radiotherapy [160].

\subsection{Cold physical plasma using inhibitor HSP90 PU-H71}

HSP90 is molecular chaperone that is expressed by ubiquitous. Disturbing its activity results in decrease tumor growth and apoptosis of cancer cells. A novel tumor therapy approach treats tumor cells with cold physical plasma which results in breakdown of HSP90 by the production of ROS. This cleavage is followed by degradation of tumor growth and angiogenesis regulator, PKD2. Cancer cells are treated with HSP90 inhibitor such as PU-H71 followed by treatment with coldplasma effects the viability of cancer cells. Thus, the synergic treatment of the two at subliminal dose is more effective. Some reports suggest the formation of ROS during cold plasma therapy induce apoptosis and arrest the cell cycle of cancer cells. Cold plasma therapy mediates molecular signals in epithelial tumors using HSP90 and its regulator PKD2. The dose of $1 \mu \mathrm{M}$ PU-H71 is adequate to induce apoptosis due to HSP90 inhibition-triggered PKD2 degradation. Using different doses to induce cancer cell death, it was found that at $50 \mathrm{nM}$ concentration cancer cells were sensitized to therapy and this dose is sufficient to benefit from synergic effect of the two treatments. Thus targeting HSP90 in combination therapy is a novel approach for treatment of cancer.[161]

\subsection{Magnetic Nanoparticles}

\subsubsection{Upregulation of Galectin-1 using Magnetic NPs}

Magnetic NP has been used for the treatment of PC [162-164] Magnetic NP that are biodegradable are prepared using recombinant human serum albumin (rHSA) and incorporated iron oxide (maghemite, $\gamma-\mathrm{Fe} 2 \mathrm{O} 3$ ) nanoparticles . Galectin-1 is a protein that is found to be upregulated in PC and its precursor lesions. Tissue plasminogen activator derived peptides (t-PA-ligands), that have 
a high affinity to galectin- 1 have been chosen as target moieties and were covalently attached onto the nanoparticle surface. Improved targeting and imaging properties were shown in mice using single photon emission computed tomography-computer tomography (SPECT-CT), a handheld gamma camera, and magnetic resonance imaging (MRI).The results were found to be promising as the NP was effective in regulating the upregulation of Galectin-1 [165].

\subsubsection{Engineered uPAR-targeted IONPs carrying chemotherapy drug gemcitabine (Gem)}

The tumor resists drug by changing its microenvironment which consist of various components such as fibroblast ,extracellular matrix, immune cells etc. [166, 167] This tumor stroma in human cancers significantly limits the delivery of therapeutic agents into cancer cells. An engineered urokinase plasminogen activator receptor (UPAR)-targeted magnetic iron oxide nanoparticles (IONPs) carrying chemotherapy drug gemcitabine (Gem) for targeted delivery into uPARexpressing tumor and stromal cells was developed. The uPARtargeted nanoparticle construct, ATF-IONP-Gem, was prepared by conjugating IONPs with the amino-terminal fragment (ATF) peptide of the receptor-binding domain of UPA, a natural ligand of uPAR, and Gem via lysosomally cleavable tetrapeptide linker. These theragnostic nanoparticles enable intracellular release of Gem following receptor-mediated endocytosis of ATF-IONP-Gem into tumor cells and also provide contrast enhancement in magnetic resonance imaging (MRI) of tumors. The study suggested that the $\mathrm{pH}$ - and lysosomal enzyme-dependent release of gemcitabine, preventing the drug from enzymatic degradation. Systemic administrations of ATF-IONP-Gem significantly inhibited the growth of orthotopic human PC xenografts in nude mice. With MRI contrast enhancement by IONPs, detected the presence of IONPs in the residual tumors following the treatment, suggesting the possibility of monitoring drug delivery and assessing drug-resistant tumors by MRI. The theranostic ATF-IONP-Gem nanoparticle has great potential for the development of targeted therapeutic and imaging approaches that are capable of overcoming the tumor stromal barrier, thus enhancing the therapeutic effect of nanoparticle drugs on pancreatic cancers.[168]

\subsubsection{Localized hyperthermia treatment of PC using magnetic nanoparticles}

Using magnetic nanoparticles to absorb alternating magnetic field energy as a method of generating localized hyperthermia has been shown to be a potential cancer treatment. This report demonstrates a system that uses tumor homing cells to actively carry iron/iron oxide nanoparticles 
into tumor tissue for alternating magnetic field treatment. Paramagnetic iron/ iron oxide nanoparticles were synthesized and loaded into RAW264.7 cells (mouse monocyte/ macrophagelike cells), which have been shown to be tumor homing cells. A murine model of disseminated peritoneal pancreatic cancer was then generated by intraperitoneal injection of Pan02 cells. After tumor development, monocyte/macrophage-like cells loaded with iron/ iron oxide nanoparticles were injected intraperitoneally and allowed to migrate into the tumor. Three days after injection, mice were exposed to an alternating magnetic field for 20 minutes to cause the cell-delivered nanoparticles to generate heat. This treatment regimen was repeated three times. A survival study demonstrated that this system can significantly increase survival in a murine pancreatic cancer model, with an average post-tumor insertion life expectancy increase of $31 \%$. This system has the potential to become a useful method for specifically and actively delivering nanoparticles for local hyperthermia treatment of cancer[169]

\subsection{Hyperthermia}

One other treatment suggested for PC cells is raising the tumor temperature (i.e., hyperthermia) by different means to kill proliferating cancer cells. Magnetization reversal process of iron oxide magnetic nanoparticles (MNPs) has enabled it to be successfully used as a heating mediator. The impact of applied heat has effect on proteins and DNA stability with ROS reported to be generated due to which induce apoptosis. [170]

Radiofrequency ablation (RFA) is the most commonly used method to treat cancers such as bone, kidney, lung brain etc however its use in pancreatic cancer is associated with problems such as injury during thermal treatment to normal pancreatic tissues which can lead to diseases such as pancreatic fistula etc. A study was performed to determine the effect of RFA in patients with unresectable PC. The procedure was performed on patients and the patients were assessed afterwards. The complication rate was found to be $24 \%$ with mortality rate of $2 \%$.Therefore the method has been reported to be effective in treatment of unresectable pancreatic cancer. [171]

There has been some other reports suggesting using RFA can be effective and safe in treatment of PC with a decrease in tumor density observed and increase survival rate of patients $[172,173]$

\subsection{Non-equilibrium atmospheric pressure plasma (NEAPP)}


Non-equilibrium atmospheric pressure plasma (NEAPP), also known as cold plasma or nonthermal atmospheric pressure plasma has been used for treatment of cancer. Plasma display anticancer activity through ROS induced apoptosis. A recent report suggests its use as an anticancer treatment method for pancreatic cancer. Mice animal model was used where they were grouped into control and PAM treated groups. Four cancer cell lines were assay using cell proliferation assay. The cancer cells were killed upon treatment. The mechanism involved may be due to apoptosis caused by PAM treatment could be induced by the production of ROS. Using optimum conditions PAM displayed selective anticancer activity against PCCs and could be considered for its treatment.[174]

\section{Conclusion}

This review gives a brief understanding of the basic pathways and major mutations that are observed in pancreatic cancer. Various approaches have been suggested by different research groups that have targeted the signaling pathways or regulating the expression of genes and growth factors along with several other treatment options that includes hyperthermia and NEAPP. The diversity of these treatment options has yet not put an end to try and develop more novel and effective drug that can target the malignant pancreatic cancer. However the heterogeneity and plasticity of the tumor itself, the invasive and the migration nature of the tumor along with late prognosis and the ability to reoccur, the lack of diagnosis tests and the less number of biomarkers available make this type of cancer the most dangerous. The review was an effort to give an overall view of the recent advances that has been made to treat the pancreatic cancer and will help the scientists to develop the more potent drugs by looking at the holistic treatment options that are so far available which will help them synthesize drugs that can eventually increase the survival rate of patients and improve the quality of life.

\section{Conflict of interest}

Authors share no conflict of interest

\section{Acknowledgment}

I would like thank islamia college Peshawar for providing necessary facilities.

\section{Funding source}


This research did not receive any specific grant from funding agencies in the public, commercial, or not-for-profit sectors.

1. Patra, K.C., et al., Mutant GNAS drives pancreatic tumourigenesis by inducing PKA-mediated SIK suppression and reprogramming lipid metabolism. Nature cell biology, 2018. 20(7): p. 811.

2. Seo, Y.D., et al., Mobilization of CD8+ T cells via CXCR4 blockade facilitates PD-1 checkpoint therapy in human pancreatic cancer. Clinical Cancer Research, 2019: p. clincanres. 0081.2019.

3. Xu, Q., et al., Stromal-derived factor-1 $\alpha / C X C L 12-C X C R 4$ chemotactic pathway promotes perineural invasion in pancreatic cancer. Oncotarget, 2015. 6(7): p. 4717.

4. Huang, J.L., et al., 519. Intravenous Application of CXCR4 Targeted Conditionally Replicative Adenovirus with Fiber and Hexon Modifications to Pancreatic Cancer. Molecular Therapy, 2016. 24: p. S207.

5. Zheng, J., et al., Extracellular matrix proteins and carcinoembryonic antigen-related cell adhesion molecules characterize pancreatic duct fluid exosomes in patients with pancreatic cancer. Hpb, 2018. 20(7): p. 597-604.

6. Jiao, Y., et al., DAXX/ATRX, MEN1, and mTOR pathway genes are frequently altered in pancreatic neuroendocrine tumors. Science, 2011. 331(6021): p. 1199-1203.

7. Lopez, C.L., et al., Chemoprevention with Somatuline (C) Delays the Progression of Pancreatic Neuroendocrine Neoplasms in a Mouse Model of Multiple Endocrine Neoplasia Type 1 (MEN1). World journal of surgery, 2019. 43(3): p. 831-838.

8. Shahbaz, M., et al., mRNA expression of somatostatin receptor subtypes SSTR-2, SSTR-3, and SSTR5 and its significance in pancreatic cancer. World journal of surgical oncology, 2015. 13(1): p. 46.

9. Morimoto, M., et al., Enhancement of the CXCL12/CXCR4 axis due to acquisition of gemcitabine resistance in pancreatic cancer: effect of CXCR4 antagonists. BMC cancer, 2016. 16(1): p. 305.

10. Sullivan, K.M., et al., Abstract A156: PDA tumor cell death as following combination anti-PD-1 blockade and CXCR4 blockade is a direct effect of CD8+ T-cells. 2019, AACR.

11. Yi, Q., et al., PRSS1 mutations and the proteinase/antiproteinase imbalance in the pathogenesis of pancreatic cancer. Tumor Biology, 2016. 37(5): p. 5805-5810.

12. Liu, C., et al., Involvement of ephrin receptor A4 in pancreatic cancer cell motility and invasion. Oncology letters, 2014. 7(6): p. 2165-2169.

13. Schnepp, R.W., et al., Mutation of tumor suppressor gene Men1 acutely enhances proliferation of pancreatic islet cells. Cancer Research, 2006. 66(11): p. 5707-5715.

14. Sierzega, M., et al., Semiquantitative immunohistochemistry for mucin (MUC1, MUC2, MUC3, MUC4, MUC5AC, and MUC6) profiling of pancreatic ductal cell adenocarcinoma improves diagnostic and prognostic performance. Histopathology, 2016. 69(4): p. 582-591.

15. Lee, J., et al., Farnesoid $X$ receptor, overexpressed in pancreatic cancer with lymph node metastasis promotes cell migration and invasion. British journal of cancer, 2011. 104(6): p. 1027.

16. Hornick, J.R., et al., The novel sigma-2 receptor ligand SW43 stabilizes pancreas cancer progression in combination with gemcitabine. Molecular cancer, 2010. 9(1): p. 298.

17. Ripka, S., et al., Glutamate receptor GRIA3-target of CUX1 and mediator of tumor progression in pancreatic cancer. Neoplasia, 2010. 12(8): p. 659-IN6.

18. Beatty, G.L., et al., Activity of mesothelin-specific chimeric antigen receptor $T$ cells against pancreatic carcinoma metastases in a phase 1 trial. Gastroenterology, 2018. 155(1): p. 29-32.

19. Panieri, E. and M. Santoro, ROS homeostasis and metabolism: a dangerous liason in cancer cells. Cell death \& disease, 2016. 7(6): p. e2253. 
20. Klaiber, U., et al., Prognostic Factors of Survival After Neoadjuvant Treatment and Resection for Initially Unresectable Pancreatic Cancer. Annals of surgery, 2019.

21. Dimitrov, D., et al., Clinical safety of focused ultrasound surgery in the treatment of advanced pancreatic cancer patients-single center prospective study. Journal of IMAB-Annual Proceeding Scientific Papers, 2019. 25(1): p. 2384-2389.

22. Watanabe, K., et al., Pancreatic cancer therapy with combined mesothelin-redirected chimeric antigen receptor T cells and cytokine-armed oncolytic adenoviruses. JCI insight, 2018. 3(7).

23. Beatty, G.L., et al., Safety and antitumor activity of chimeric antigen receptor modified T cells in patients with chemotherapy refractory metastatic pancreatic cancer. 2015, American Society of Clinical Oncology.

24. Rudra, S., et al., Using adaptive magnetic resonance image-guided radiation therapy for treatment of inoperable pancreatic cancer. Cancer medicine, 2019.

25. Springfeld, C., et al., Chemotherapy for pancreatic cancer. La Presse Médicale, 2019.

26. Park, S.J. and M.A. Lee, Oral chemotherapy as second-line treatment option for gemcitabinerefractory advanced pancreatic cancer with poor performance status. 2019, American Society of Clinical Oncology.

27. Lei, F., et al., Combination Therapies and Drug Delivery Platforms in Combating Pancreatic Cancer. The Journal of pharmacology and experimental therapeutics, 2019.

28. Neesse, A., et al., Stromal biology and therapy in pancreatic cancer: ready for clinical translation? Gut, 2019. 68(1): p. 159-171.

29. Clement, S., et al. Nuclear targeted x-ray activated photodynamic therapy: a solution to treat pancreatic cancer (Conference Presentation). in Optical Methods for Tumor Treatment and Detection: Mechanisms and Techniques in Photodynamic Therapy XXVIII. 2019. International Society for Optics and Photonics.

30. Nakaizumi, A., et al., Diagnosis of pancreatic cancer by cytology and measurement of oncogene and tumor markers in pure pancreatic juice aspirated by endoscopy. Hepato-gastroenterology, 1999. 46(25): p. 31-37.

31. Neoptolemos, J.P., et al., Apoptosis signaling pathways in pancreatic cancer pathogenesis. Pancreatic Cancer, 2010: p. 369-386.

32. Yu, W., et al., Chronic ethanol exposure of human pancreatic normal ductal epithelial cells induces cancer stem cell phenotype through SATB2. Journal of cellular and molecular medicine, 2018. 22(8): p. 3920-3928.

33. Shrihari, T., Dual role of inflammatory mediators in cancer. Ecancermedicalscience, 2017. 11.

34. Butler, A.E., et al., Marked expansion of exocrine and endocrine pancreas with incretin therapy in humans with increased exocrine pancreas dysplasia and the potential for glucagon-producing neuroendocrine tumors. Diabetes, 2013. 62(7): p. 2595-2604.

35. Cloer, E.W., et al., NRF2 Activation in Cancer: From DNA to Protein. Cancer research, 2019. 79(5): p. 889-898.

36. Oeckinghaus, A. and S. Ghosh, The NF-KB family of transcription factors and its regulation. Cold Spring Harbor perspectives in biology, 2009. 1(4): p. a000034.

37. He, T.-C., et al., Identification of c-MYC as a target of the APC pathway. Science, 1998. 281(5382): p. $1509-1512$.

38. Liang, C., et al., PIN1 Maintains Redox Balance via the c-Myc/NRF2 Axis to Counteract Kras-Induced Mitochondrial Respiratory Injury in Pancreatic Cancer Cells. Cancer Research, 2019. 79(1): p. 133145.

39. Owusu-Brackett, N., M. Shariati, and F. Meric-Bernstam, Role of PI3K/AKT/mTOR in Cancer Signaling, in Predictive Biomarkers in Oncology. 2019, Springer. p. 263-270. 
40. Di Domenico, F., et al., Restoration of aberrant mTOR signaling by intranasal rapamycin reduces oxidative damage: Focus on HNE-modified proteins in a mouse model of down syndrome. Redox biology, 2019: p. 101162.

41. Ozawa, F., et al., Growth factors and their receptors in pancreatic cancer. Teratogenesis, carcinogenesis, and mutagenesis, 2001. 21(1): p. 27-44.

42. Smith, J.P., L.K. Fonkoua, and T.W. Moody, The role of gastrin and CCK receptors in pancreatic cancer and other malignancies. International journal of biological sciences, 2016. 12(3): p. 283.

43. Costache, M., et al., VEGF expression in pancreatic cancer and other malignancies: a review of the literature. Romanian journal of internal medicine, 2015. 53(3): p. 199-208.

44. Hogendorf, P., et al., Pancreatic head carcinoma and vascular endothelial growth factor (VEGF-A) concentration in portal blood: its association with cancer grade, tumor size and probably poor prognosis. Archives of medical science: AMS, 2014. 10(2): p. 288.

45. Pysz, M.A., et al., Vascular endothelial growth factor receptor type 2-targeted contrast-enhanced US of pancreatic cancer neovasculature in a genetically engineered mouse model: potential for earlier detection. Radiology, 2014. 274(3): p. 790-799.

46. Yao, $\mathrm{H}_{\text {., }}$ et al., Role of $\alpha 561$ integrin up-regulation in radiation-induced invasion by human pancreatic cancer cells. Translational oncology, 2011. 4(5): p. 282-292.

47. Li, M., et al., Somatostatin, somatostatin receptors, and pancreatic cancer. World journal of surgery, 2005. 29(3): p. 293-296.

48. Annese, T., et al., Angiogenesis in Pancreatic Cancer: Pre-Clinical and Clinical Studies. Cancers, 2019. 11(3): p. 381.

49. Pineda, D.M., et al., HuR's post-transcriptional regulation of Death Receptor 5 in pancreatic cancer cells. Cancer biology \& therapy, 2012. 13(10): p. 946-955.

50. Kojima, T., D. Kyuno, and N. Sawada, Targeting claudin-4 in human pancreatic cancer. Expert opinion on therapeutic targets, 2012. 16(9): p. 881-887.

51. Michl, P., et al., Claudin-4: a new target for pancreatic cancer treatment using Clostridium perfringens enterotoxin. Gastroenterology, 2001. 121(3): p. 678-684.

52. Nichols, L.S., R. Ashfaq, and C.A. lacobuzio-Donahue, Claudin 4 protein expression in primary and metastatic pancreatic cancer: support for use as a therapeutic target. American journal of clinical pathology, 2004. 121(2): p. 226-230.

53. Damaskos, C., et al., Nuclear receptors in pancreatic tumor cells. Anticancer research, 2014. 34(12): p. 6897-6911.

54. Seeliger, H., et al., Expression of estrogen receptor beta correlates with adverse prognosis in resected pancreatic adenocarcinoma. BMC cancer, 2018. 18(1): p. 1049.

55. Li, L., et al., Antibody against CD44s inhibits pancreatic tumor initiation and postradiation recurrence in mice. Gastroenterology, 2014. 146(4): p. 1108-1118. e12.

56. Ryschich, E., et al., Transferrin receptor is a marker of malignant phenotype in human pancreatic cancer and in neuroendocrine carcinoma of the pancreas. European journal of cancer, 2004. 40(9): p. 1418-1422.

57. Jeong, S.M., S. Hwang, and R.H. Seong, Transferrin receptor regulates pancreatic cancer growth by modulating mitochondrial respiration and ROS generation. Biochemical and biophysical research communications, 2016. 471(3): p. 373-379.

58. Ramachandran, V., et al., Adrenomedullin is expressed in pancreatic cancer and stimulates cell proliferation and invasion in an autocrine manner via the adrenomedullin receptor, ADMR. Cancer research, 2007. 67(6): p. 2666-2675.

59. Grover, P., MUC1 Mediated Signaling in Pancreatic Ductal Adenocarcinoma. 2019, The University of North Carolina at Charlotte. 
60. Chia, L.A. and C.J. Kuo, The intestinal stem cell, in Progress in molecular biology and translational science. 2010, Elsevier. p. 157-173.

61. Navas, C., et al., EGF receptor signaling is essential for k-ras oncogene-driven pancreatic ductal adenocarcinoma. Cancer cell, 2012. 22(3): p. 318-330.

62. Dosch, A.R., et al., Src kinase inhibition restores E-cadherin expression in dasatinib-sensitive pancreatic cancer cells. Oncotarget, 2019. 10(10): p. 1056.

63. Deng, S.-J., et al., Nutrient Stress-Dysregulated Antisense IncRNA GLS-AS Impairs GLS-Mediated Metabolism and Represses Pancreatic Cancer Progression. Cancer research, 2019. 79(7): p. 13981412.

64. Wang, G., et al., Serine/arginine protein-specific kinase 2 promotes the development and progression of pancreatic cancer by downregulating Numb and p53. The FEBS journal, 2019.

65. Wu, S.-Z., et al., Dihydrosanguinarine suppresses pancreatic cancer cells via regulation of mutp53/WT-p53 and the Ras/Raf/Mek/Erk pathway. Phytomedicine, 2019: p. 152895.

66. Jia, Y., et al., The role of GLI-SOX2 signaling axis for gemcitabine resistance in pancreatic cancer. Oncogene, 2019. 38(10): p. 1764.

67. Hu, C., et al., Association between inherited germline mutations in cancer predisposition genes and risk of pancreatic cancer. Jama, 2018. 319(23): p. 2401-2409.

68. Klein, A.P., Genetic susceptibility to pancreatic cancer. Molecular carcinogenesis, 2012. 51(1): p. 14-24.

69. Funamizu, N., et al., MicroRNA-200b and-301 are associated with gemcitabine response as biomarkers in pancreatic carcinoma cells. International journal of oncology, 2019. 54(3): p. 9911000.

70. Ozaki, T., et al., Impact of RUNX2 on drug-resistant human pancreatic cancer cells with p53 mutations. BMC cancer, 2018. 18(1): p. 309.

71. Krebs, A.M., et al., The EMT-activator Zeb1 is a key factor for cell plasticity and promotes metastasis in pancreatic cancer. Nature cell biology, 2017. 19(5): p. 518.

72. Cazacu, I.M., et al., Pancreatitis-Associated Genes and Pancreatic Cancer Risk: A Systematic Review and Meta-analysis. Pancreas, 2018. 47(9): p. 1078.

73. Vincent, A., et al., Pancreatic cancer. The lancet, 2011. 378(9791): p. 607-620.

74. Hidalgo, M., Pancreatic cancer. New England Journal of Medicine, 2010. 362(17): p. 1605-1617.

75. Loosen, S.H., et al., High baseline soluble urokinase plasminogen activator receptor (suPAR) serum levels indicate adverse outcome after resection of pancreatic adenocarcinoma. Carcinogenesis, 2019.

76. Ding, Y. and Y. Du, Clinicopathological significance and prognostic role of chemokine receptor CXCR4 expression in pancreatic ductal adenocarcinoma, a meta-analysis and literature review. International Journal of Surgery, 2019.

77. Ahmed, S., et al., The TGF-8/Smad4 signaling pathway in pancreatic carcinogenesis and its clinical significance. Journal of clinical medicine, 2017. 6(1): p. 5.

78. Pommier, Y., et al., Apoptosis defects and chemotherapy resistance: molecular interaction maps and networks. Oncogene, 2004. 23(16): p. 2934.

79. Westphal, S. and H. Kalthoff, Apoptosis: targets in pancreatic cancer. Molecular cancer, 2003. 2(1): p. 6.

80. Mcllwain, D.R., T. Berger, and T.W. Mak, Caspase functions in cell death and disease. Cold Spring Harbor perspectives in biology, 2013. 5(4): p. a008656.

81. Modi, S., et al., Control of apoptosis in treatment and biology of pancreatic cancer. Journal of cellular biochemistry, 2016. 117(2): p. 279-288.

82. Sano, R. and J.C. Reed, ER stress-induced cell death mechanisms. Biochimica et Biophysica Acta (BBA)-Molecular Cell Research, 2013. 1833(12): p. 3460-3470. 
83. Elmore, S., Apoptosis: a review of programmed cell death. Toxicologic pathology, 2007. 35(4): $p$. 495-516.

84. Xiong, S., et al., Mitochondria-mediated apoptosis in mammals. Protein \& cell, 2014. 5(10): p. 737749.

85. Fulda, S. and K.-M. Debatin, Extrinsic versus intrinsic apoptosis pathways in anticancer chemotherapy. Oncogene, 2006. 25(34): p. 4798.

86. Gu, D., K.E. Schlotman, and J. Xie, Deciphering the role of hedgehog signaling in pancreatic cancer. Journal of biomedical research, 2016. 30(5): p. 353.

87. Bai, Y., et al., Hedgehog signaling in pancreatic fibrosis and cancer. Medicine, 2016. 95(10).

88. Fu, J., et al., Triacetyl resveratrol upregulates miRNA-200 and suppresses the Shh pathway in pancreatic cancer: A potential therapeutic agent. International journal of oncology, 2019. 54(4): p. 1306-1316.

89. Cardama, G., et al., Rho GTPases as therapeutic targets in cancer. International journal of oncology, 2017. 51(4): p. 1025-1034.

90. Yan, Y. and M.M. Ouellette, Rac1 GTPase in pancreatic cancer. Aging (Albany NY), 2015. 7(9): p. 609.

91. Eser, S., et al., Oncogenic KRAS signalling in pancreatic cancer. British journal of cancer, 2014. 111(5): p. 817.

92. Neuzillet, C., et al., Targeting the TGFB pathway for cancer therapy. Pharmacology \& therapeutics, 2015. 147: p. 22-31.

93. Melisi, D., et al., TGFB receptor inhibitor galunisertib is linked to inflammation-and remodelingrelated proteins in patients with pancreatic cancer. Cancer chemotherapy and pharmacology, 2019: p. 1-17.

94. Lambies, G., et al., TGFB-activated USP27X deubiquitinase regulates cell migration and chemoresistance via stabilization of Snail1. Cancer research, 2019. 79(1): p. 33-46.

95. Principe, D.R., et al., TGFB Blockade Augments PD-1 Inhibition to Promote T-Cell-Mediated Regression of Pancreatic Cancer. Molecular cancer therapeutics, 2019. 18(3): p. 613-620.

96. Ristorcelli, E. and D. Lombardo, Targeting Notch signaling in pancreatic cancer. Expert opinion on therapeutic targets, 2010. 14(5): p. 541-552.

97. Nawroth, R., et al., Extracellular sulfatases, elements of the Wnt signaling pathway, positively regulate growth and tumorigenicity of human pancreatic cancer cells. PloS one, 2007. 2(4): p. e392.

98. Wang, Z., et al., Blockade of SDF-1/CXCR4 signalling inhibits pancreatic cancer progression in vitro via inactivation of canonical Wnt pathway. British journal of cancer, 2008. 99(10): p. 1695.

99. Wang, L., et al., Oncogenic function of ATDC in pancreatic cancer through Wht pathway activation and 8-catenin stabilization. Cancer cell, 2009. 15(3): p. 207-219.

100. Li, W., et al., Growth of the pancreatic cancer cell line PANC-1 is inhibited by protein phosphatase $2 A$ inhibitors through overactivation of the c-Jun N-terminal kinase pathway. European journal of cancer, 2011. 47(17): p. 2654-2664.

101. Mantoni, T.S., et al., Pancreatic stellate cells radioprotect pancreatic cancer cells through 61 integrin signaling. Cancer research, 2011. 71(10): p. 3453-3458.

102. Ji, Z., et al., Oncogenic KRAS activates hedgehog signaling pathway in pancreatic cancer cells. Journal of Biological Chemistry, 2007. 282(19): p. 14048-14055.

103. Zhang, Y., et al., cordycepin induces apoptosis in human pancreatic cancer cells via the mitochondrial-mediated intrinsic pathway and suppresses tumor growth in vivo. OncoTargets and therapy, 2018. 11: p. 4479.

104. Okura, R., et al., MicroRNA profiles during galectin-9-induced apoptosis of pancreatic cancer cells. Oncology letters, 2018. 15(1): p. 407-414. 
105. Mocellin, S. and M. Provenzano, RNA interference: learning gene knock-down from cell physiology. Journal of translational medicine, 2004. 2(1): p. 39.

106. Lin, S., et al., Involvement of endoplasmic reticulum stress in capsaicin-induced apoptosis of human pancreatic cancer cells. Evidence-Based Complementary and Alternative Medicine, 2013. 2013.

107. Li, X., et al., Combined inhibition of autophagy and Nrf2 signaling augments bortezomib-induced apoptosis by increasing ROS production and ER stress in pancreatic cancer cells. International journal of biological sciences, 2018. 14(10): p. 1291.

108. White, M.C., et al., HRI-mediated translational repression reduces proteotoxicity and sensitivity to bortezomib in human pancreatic cancer cells. Oncogene, 2018. 37(32): p. 4413.

109. Hu, Y., et al., A novel STAT3 inhibitor HO-3867 induces cell apoptosis by reactive oxygen speciesdependent endoplasmic reticulum stress in human pancreatic cancer cells. Anti-cancer drugs, 2017. 28(4): p. 392-400.

110. Li, Z., CD133: a stem cell biomarker and beyond. Experimental hematology \& oncology, 2013. 2(1): p. 17.

111. Yoshida, G.J., Metabolic reprogramming: the emerging concept and associated therapeutic strategies. Journal of experimental \& clinical cancer research, 2015. 34(1): p. 111.

112. Ward, P.S. and C.B. Thompson, Metabolic reprogramming: a cancer hallmark even warburg did not anticipate. Cancer cell, 2012. 21(3): p. 297-308.

113. Dauer, P., et al., ER stress sensor, glucose regulatory protein 78 (GRP78) regulates redox status in pancreatic cancer thereby maintaining "stemness". Cell death \& disease, 2019. 10(2): p. 132.

114. Mao, Y., et al., Regulation of cell apoptosis and proliferation in pancreatic cancer through PI3K/Akt pathway via Polo-like kinase 1. Oncology reports, 2016. 36(1): p. 49-56.

115. Luo, J., et al., High Glucose-Induced ROS Production Stimulates Proliferation of Pancreatic Cancer via Inactivating the JNK Pathway. Oxidative medicine and cellular longevity, 2018. 2018.

116. Suzuki, S., et al., JNK suppression of chemotherapeutic agents-induced ROS confers chemoresistance on pancreatic cancer stem cells. Oncotarget, 2015. 6(1): p. 458.

117. Wang, G., et al., ROS mediated EGFR/MEK/ERK/HIF-1 $\alpha$ Loop Regulates Glucose metabolism in pancreatic cancer. Biochemical and Biophysical Research Communications, 2018. 500(4): p. 873878.

118. Xu, Z., et al., Sophoridine induces apoptosis and S phase arrest via ROS-dependent JNK and ERK activation in human pancreatic cancer cells. Journal of Experimental \& Clinical Cancer Research, 2017. 36(1): p. 124.

119. Zhang, R., et al., In vitro and in vivo induction of apoptosis by capsaicin in pancreatic cancer cells is mediated through ROS generation and mitochondrial death pathway. Apoptosis, 2008. 13(12): p. 1465-1478.

120. Meng, Q., et al., Abrogation of glutathione peroxidase-1 drives EMT and chemoresistance in pancreatic cancer by activating ROS-mediated Akt/GSK36/snail signaling. Oncogene, 2018. 37(44): p. 5843.

121. Zhang, Z., et al., Gemcitabine treatment promotes pancreatic cancer stemness through the Nox/ROS/NF-KB/STAT3 signaling cascade. Cancer letters, 2016. 382(1): p. 53-63.

122. Abdalla, M.Y., et al., Enhancing responsiveness of pancreatic cancer cells to gemcitabine treatment under hypoxia by heme oxygenase-1 inhibition. Translational Research, 2019.

123. Han, L., et al., The inhibition of heme oxygenase-1 enhances the chemosensitivity and suppresses the proliferation of pancreatic cancer cells through the SHH signaling pathway. International journal of oncology, 2018. 52(6): p. 2101-2109.

124. Hu, Q., et al., UHRF1 promotes aerobic glycolysis and proliferation via suppression of SIRT4 in pancreatic cancer. Cancer Letters, 2019. 
125. Huang, G. and G. Zhu, Sirtuin-4 (SIRT4), a therapeutic target with oncogenic and tumorsuppressive activity in cancer. OncoTargets and therapy, 2018. 11: p. 3395.

126. Hu, Q., et al., $d C K$ negatively regulates the NRF 2/ARE axis and ROS production in pancreatic cancer. Cell Proliferation, 2018. 51(4): p. e12456.

127. Lister, A., et al., Nrf2 is overexpressed in pancreatic cancer: implications for cell proliferation and therapy. Molecular cancer, 2011. 10(1): p. 37.

128. Gambardella, V., et al., NRF2 through RPS6 activation is related to anti-HER2 drug resistance in HER2-amplified gastric Cancer. Clinical Cancer Research, 2019. 25(5): p. 1639-1649.

129. Leung, C.H., et al., Nuclear Factor (Erythroid-Derived 2)-Like 2 Regulates the Hepatoprotective Effects of Remote Ischemic Conditioning in Hemorrhagic Shock. Antioxidants \& Redox Signaling.

130. Javle, M., et al., Biomarkers of TGF-B signaling pathway and prognosis of pancreatic cancer. PloS one, 2014. 9(1): p. e85942.

131. Jakowlew, S.B., Transforming growth factor- 8 in cancer and metastasis. Cancer and Metastasis Reviews, 2006. 25(3): p. 435.

132. Truty, M.J. and R. Urrutia, Basics of TGF-B and pancreatic cancer. Pancreatology, 2007. 7(5-6): $p$. 423-435.

133. Fabregat, I., et al., TGF-beta signaling in cancer treatment. Current pharmaceutical design, 2014. 20(17): p. 2934-2947.

134. Shen, W., et al., TGF-B in pancreatic cancer initiation and progression: two sides of the same coin. Cell \& bioscience, 2017. 7(1): p. 39.

135. Principe, D.R., et al., TGFB signaling in the pancreatic tumor microenvironment promotes fibrosis and immune evasion to facilitate tumorigenesis. Cancer research, 2016. 76(9): p. 2525-2539.

136. Grover, P., et al., SMAD4-independent activation of TGF-B signaling by MUC1 in a human pancreatic cancer cell line. Oncotarget, 2018. 9(6): p. 6897.

137. Zhou, Y., et al., Digoxin sensitizes gemcitabine-resistant pancreatic cancer cells to gemcitabine via inhibiting Nrf2 signaling pathway. Redox biology, 2019. 22: p. 101131.

138. Yan, Y., et al., Active ingredients of Inula helenium L. exhibits similar anti-cancer effects as isoalantolactone in pancreatic cancer cells. Natural product research, 2019: p. 1-6.

139. Tebay, L.E., et al., Mechanisms of activation of the transcription factor Nrf2 by redox stressors, nutrient cues, and energy status and the pathways through which it attenuates degenerative disease. Free Radical Biology and Medicine, 2015. 88: p. 108-146.

140. DeNicola, G.M., et al., Oncogene-induced Nrf2 transcription promotes ROS detoxification and tumorigenesis. Nature, 2011. 475(7354): p. 106.

141. Canning, P., F.J. Sorrell, and A.N. Bullock, Structural basis of Keap1 interactions with Nrf2. Free Radical Biology and Medicine, 2015. 88: p. 101-107.

142. Schmoll, D., C.K. Engel, and H. Glombik, The Keap1-Nrf2 protein-protein interaction: A suitable target for small molecules. Drug Discovery Today: Technologies, 2017. 24: p. 11-17.

143. Yoshizaki, Y., et al., Drug-repositioning screening for Keap1-Nrf2 binding inhibitors using fluorescence correlation spectroscopy. Scientific reports, 2017. 7(1): p. 3945.

144. Karttunen, M., W.-Y. Choy, and E.A. Cino, Prediction of Binding Energy of Keap1 Interaction Motifs in the Nrf2 Antioxidant Pathway and Design of Potential High-Affinity Peptides. The Journal of Physical Chemistry B, 2018. 122(22): p. 5851-5859.

145. Cheng, L., et al., Resveratrol-induced downregulation of NAF-1 enhances the sensitivity of pancreatic cancer cells to gemcitabine via the ROS/Nrf2 signaling pathways. Oxidative medicine and cellular longevity, 2018. 2018.

146. Yoon, K.-A., et al., Comprehensive Cancer Panel Sequencing Defines Genetic Diversity and Changes in the Mutational Characteristics of Pancreatic Cancer Patients Receiving Neoadjuvant Treatment. Gut and liver, 2019. 
147. Vicente-Duenas, C., et al., The role of cellular plasticity in cancer development. Current medicinal chemistry, 2009. 16(28): p. 3676-3685.

148. Crawford, H.C., M.P. di Magliano, and S. Banerjee, Signaling Networks that Control Cellular Plasticity in Pancreatic Tumorigenesis, Progression and Metastasis. Gastroenterology, 2019.

149. Storz, P., KRas, ROS and the initiation of pancreatic cancer. Small GTPases, 2017. 8(1): p. 38-42.

150. Tirella, A., et al., CD44 targeted delivery of siRNA by using HA-decorated nanotechnologies for KRAS silencing in cancer treatment. International journal of pharmaceutics, 2019. 561: p. 114-123.

151. Concepcion, C.P., C. Bonetti, and A. Ventura, The miR-17-92 family of microRNA clusters in development and disease. Cancer journal (Sudbury, Mass.), 2012. 18(3): p. 262.

152. Bos, J.L., The ras gene family and human carcinogenesis. Mutation Research/Reviews in Genetic Toxicology, 1988. 195(3): p. 255-271.

153. Bryant, K.L., et al., KRAS: feeding pancreatic cancer proliferation. Trends in biochemical sciences, 2014. 39(2): p. 91-100.

154. Yu, S., et al., miRNA-96 suppresses KRAS and functions as a tumor suppressor gene in pancreatic cancer. Cancer research, 2010. 70(14): p. 6015-6025.

155. Vorvis, C., M. Koutsioumpa, and D. Iliopoulos, Developments in miRNA gene signaling pathways in pancreatic cancer. Future Oncology, 2016. 12(9): p. 1135-1150.

156. Khvalevsky, E.Z., et al., Mutant KRAS is a druggable target for pancreatic cancer. Proceedings of the National Academy of Sciences, 2013. 110(51): p. 20723-20728.

157. Golan, T., et al., RNAi therapy targeting KRAS in combination with chemotherapy for locally advanced pancreatic cancer patients. Oncotarget, 2015. 6(27): p. 24560.

158. Yoon, Y.J., O.Y. Kim, and Y.S. Gho, Extracellular vesicles as emerging intercellular communicasomes. BMB reports, 2014. 47(10): p. 531.

159. Patel, G.K., et al., Exosomes confer chemoresistance to pancreatic cancer cells by promoting ROS detoxification and miR-155-mediated suppression of key gemcitabine-metabolising enzyme, DCK. British journal of cancer, 2017. 116(5): p. 609.

160. Wu, D.-M., et al., Bone Marrow Mesenchymal Stem Cell-Derived Exosomal microRNA-126-3p Inhibits Proliferation, Migration, Invasion and Promotes Apoptosis in Pancreatic Cancer via ADAM9. Molecular Therapy-Nucleic Acids, 2019.

161. Kamerkar, S., et al., Exosomes facilitate therapeutic targeting of oncogenic KRAS in pancreatic cancer. Nature, 2017. 546(7659): p. 498.

162. Kong, C., et al., Targeting the Oncogene KRAS Mutant Pancreatic Cancer by Synergistic Blocking of Lysosomal Acidification and Rapid Drug Release. ACS nano, 2019.

163. Yao, W., et al., Syndecan 1 is a critical mediator of macropinocytosis in pancreatic cancer. Nature, 2019: p. 1.

164. Zeitouni, D., et al., KRAS mutant pancreatic cancer: no lone path to an effective treatment. Cancers, 2016. 8(4): p. 45.

165. Bryant, K.L., et al., Combination of ERK and autophagy inhibition as a treatment approach for pancreatic cancer. Nature medicine, 2019: p. 1.

166. Seton-Rogers, S., Eliminating protective autophagy in KRAS-mutant cancers. Nature Reviews Cancer, 2019: p. 1.

167. C Prendergast, G., et al., Indoleamine 2, 3-dioxygenase as a modifier of pathogenic inflammation in cancer and other inflammation-associated diseases. Current medicinal chemistry, 2011. 18(15): p. 2257-2262.

168. Nevler, A., et al., Host IDO2 gene status influences tumor progression and radiotherapy response in KRAS-driven sporadic pancreatic cancers. Clinical Cancer Research, 2019. 25(2): p. 724-734.

169. Bekeschus, S., et al., Physical plasma-triggered ROS induces tumor cell death upon cleavage of HSP90 chaperone. Scientific reports, 2019. 9(1): p. 4112. 
170. Gang, J., et al., Magnetic poly $\varepsilon$-caprolactone nanoparticles containing Fe3O4 and gemcitabine enhance anti-tumor effect in pancreatic cancer xenograft mouse model. Journal of drug targeting, 2007. 15(6): p. 445-453.

171. Singh, A. and S.K. Sahoo, Magnetic nanoparticles: a novel platform for cancer theranostics. Drug discovery today, 2014. 19(4): p. 474-481.

172. Yang, L., et al., Molecular imaging of pancreatic cancer in an animal model using targeted multifunctional nanoparticles. Gastroenterology, 2009. 136(5): p. 1514-1525. e2.

173. Rosenberger, I., et al., Targeted diagnostic magnetic nanoparticles for medical imaging of pancreatic cancer. Journal of controlled release, 2015. 214: p. 76-84.

174. Bremnes, R.M., et al., The role of tumor stroma in cancer progression and prognosis: emphasis on carcinoma-associated fibroblasts and non-small cell lung cancer. Journal of thoracic oncology, 2011. 6(1): p. 209-217.

175. Werb, Z. and P. Lu, The role of stroma in tumor development. Cancer journal (Sudbury, Mass.), 2015. 21(4): p. 250.

176. Lee, G.Y., et al., Theranostic nanoparticles with controlled release of gemcitabine for targeted therapy and MRI of pancreatic cancer. ACS nano, 2013. 7(3): p. 2078-2089.

177. Basel, M.T., et al., Cell-delivered magnetic nanoparticles caused hyperthermia-mediated increased survival in a murine pancreatic cancer model. International journal of nanomedicine, 2012. 7: p. 297.

178. Ludwig, R., et al., Nanoparticle-based hyperthermia distinctly impacts production of ROS, expression of Ki-67, TOP2A, and TPX2, and induction of apoptosis in pancreatic cancer. International journal of nanomedicine, 2017. 12: p. 1009.

179. Girelli, R., et al., Feasibility and safety of radiofrequency ablation for locally advanced pancreatic cancer. British Journal of Surgery, 2010. 97(2): p. 220-225.

180. Spiliotis, J.D., et al., Radiofrequency ablation combined with palliative surgery may prolong survival of patients with advanced cancer of the pancreas. Langenbeck's archives of surgery, 2007. 392(1): p. 55-60.

181. Hadjicostas, P., et al., Radiofrequency ablation in pancreatic cancer. HPB, 2006. 8(1): p. 61-64.

182. Hattori, N., et al., Effectiveness of plasma treatment on pancreatic cancer cells. International journal of oncology, 2015. 47(5): p. 1655-1662. 